\title{
THE CHERN NUMBERS AND EULER CHARACTERISTICS OF MODULES
}

\author{
L. GHEZZI, S. GOTO, J. HONG, K. OZEKI, T.T. PHUONG, AND W. V. VASCONCELOS
}

\begin{abstract}
The set of the first Hilbert coefficients of parameter ideals relative to a module-its Chern coefficients-over a local Noetherian ring codes for considerable information about its structure-noteworthy properties such as that of Cohen-Macaulayness, Buchsbaumness, and of having finitely generated local cohomology. The authors have previously studied the ring case. By developing a robust setting to treat these coefficients for unmixed rings and modules, the case of modules is analyzed in a more transparent manner. Another series of integers arise from partial Euler characteristics and are shown to carry similar properties of the module. The technology of homological degree theory is also introduced in order to derive bounds for these two sets of numbers.
\end{abstract}

Dedicated to Professors N. V. Trung and G. Valla for their groundbreaking contributions to the theory of Hilbert functions.

\section{INTRODUCTION}

Let $\mathbf{R}$ be a Noetherian local ring with maximal ideal $\mathfrak{m}$ and let $I$ be an $\mathfrak{m}$-primary ideal. There is a great deal of interest on the set of $I$-good filtrations of $\mathbf{R}$. More concretely, on the set of multiplicative, decreasing filtrations

$$
\mathcal{A}=\left\{I_{n} \mid I_{0}=\mathbf{R}, I_{n+1}=I I_{n}, n \gg 0\right\}
$$

of $\mathbf{R}$ ideals which are integral over the $I$-adic filtration, conveniently coded in the corresponding Rees algebra and its associated graded ring

$$
\mathcal{R}(\mathcal{A})=\sum_{n \geq 0} I_{n} t^{n}, \quad \operatorname{gr}_{\mathcal{A}}(\mathbf{R})=\sum_{n \geq 0} I_{n} / I_{n+1} .
$$

Our focus here is on a set of filtrations both broader and more narrowly defined. Let $M$ be a finitely generated $\mathbf{R}$-module. The Hilbert polynomial of the associated graded module

$$
\operatorname{gr}_{I}(M)=\bigoplus_{n \geq 0} I^{n} M / I^{n+1} M
$$

more precisely the values of the length $\lambda\left(M / I^{n+1} M\right)$ of $M / I^{n+1} M$ for large $n$, can be assembled as

$$
P_{M}(n)=\sum_{i=0}^{r}(-1)^{i} e_{i}(I, M)\left(\begin{array}{c}
n+r-i \\
r-i
\end{array}\right),
$$

AMS 2010 Mathematics Subject Classification: 13H10, 13H15, $13 \mathrm{~A} 30$.

The first author is partially supported by a grant from the City University of New York PSC-CUNY Research Award Program-44. The second author is partially supported by Grant-in-Aid for Scientific Researches (C) in Japan (19540054). The fourth author is supported by a grant from MIMS (Meiji Institute for Advanced Study of Mathematical Sciences). The fifth author is supported by JSPS Ronpaku (Dissertation of $\mathrm{PhD})$ Program. The last author is partially supported by the NSF. 
where $r=\operatorname{dim}_{\mathbf{R}} M>0$. In most of our discussion, either $I$ or $M$ is fixed, and by simplicity we set $e_{i}(I, M)=e_{i}(M)$ or $e_{i}(I, M)=e_{i}(I)$, accordingly. Occasionally the first Hilbert coefficient $e_{1}(I, M)$ is referred to as the Chern coefficient of $I$ relative to $M$ ([32]).

The authors have examined ([8], [9, [13], 32]) how the values of $e_{1}(Q, \mathbf{R})$ codes for structural information about the ring $\mathbf{R}$ itself. More explicitly one defines the set

$$
\Lambda(M)=\left\{e_{1}(Q, M) \mid Q \text { is a parameter ideal for } M\right\}
$$

and examines what its structure expresses about $M$. In case $M=\mathbf{R}$, this set was analyzed for the following extremal properties:

(a) $0 \in \Lambda(\mathbf{R})$

(b) $\Lambda(\mathbf{R})$ contains a single element;

(c) $\Lambda(\mathbf{R})$ is bounded.

The task of determining the elements of $\Lambda(M)$ has turned out to be rather daunting. More amenable has been the approach to obtain specialized bounds using cohomological techniques. An unresolved issue has been to describe the character of the set $\Lambda(M)$, in particular the role of its extrema and the gap structure of the set itself.

The other invariant of the module $M$ in our investigation is the following. Let $Q=$ $\left(x_{1}, x_{2}, \ldots, x_{r}\right)$ be a parameter ideal for $M$. We denote by $\mathrm{H}_{i}(Q ; M)(i \in \mathbb{Z})$ the $i$-th homology module of the Koszul complex $\mathrm{K}_{\bullet}(Q ; M)$ generated by the system $\mathbf{x}=\left\{x_{1}, x_{2}, \ldots, x_{r}\right\}$ of parameters of $M$. We put

$$
\chi_{1}(Q ; M)=\sum_{i \geq 1}(-1)^{i-1} \lambda_{\mathbf{R}}\left(\mathrm{H}_{i}(Q ; M)\right)
$$

and call it the first Euler characteristic of $M$ relative to $Q$; hence

$$
\chi_{1}(Q ; M)=\lambda_{\mathbf{R}}(M / Q M)-e_{0}(Q, M)
$$

by a classical result of Serre (see [1], [26]).

In analogy to $\Lambda(M)$, one defines the set

$$
\Xi(M)=\left\{\chi_{1}(Q ; M) \mid Q \text { is a parameter ideal for } M\right\}
$$

and examines again what its structure expresses about $M$. Most of the properties of this set can be assembled from a diverse literature, particularly from [26, Appendice II]. The outcome is a listing that mirrors, step-by-step, all the properties of the set $\Lambda(M)$ that we study.

We shall now describe more precisely our results. Section 2 starts with a review of some elementary computation rules for $e_{1}(Q, M)$ under hyperplane sections, more properly modulo superficial elements. Since part of our goal is to extend to modules our previous results on rings, given the ubiquity of the unmixedness hypothesis, we develop a fresh setting to treat the module case. It made for more transparent proofs. These are carried out in Sections 3-5.

Section 6 introduces homological degree techniques to obtain special bounds for the set $\Lambda(M)$. The treatment here is more general and sharper than in [32]. Thus in Corollary 6.7 it is proved that the set

$\Lambda_{Q}(M)=\left\{e_{1}(\mathfrak{q}, M): \mathfrak{q}\right.$ is a parameter ideal for $M$ with the same integral closure as that of $\left.Q\right\}$, is finite. In Section 7, we treat the sets $\Xi(M)$ and $\Xi_{Q}(M)$, focusing on the properties that have analogs in $\Lambda(M)$ (see Table 1). In particular, we prove that Euler characteristics can 
be uniformly bounded by homological degrees (Theorem [7.2). We also consider the numerical function, which we call the Hilbert characteristic of $M$ with respect to $Q=(\mathbf{x})$ :

$$
\mathbf{h}(\mathbf{x} ; M)=\sum_{i=0}^{r}(-1)^{i} e_{i}(Q, M) .
$$

If the system $\mathbf{x}=\left\{x_{1}, x_{2}, \ldots, x_{r}\right\}$ of parameters of $M$ forms a $d$-sequence for $M, \mathbf{h}(\mathbf{x} ; M)$ has some properties of a homological degree. They are enough to bound the Betti numbers $\beta_{i}(M)$ in terms of $\beta_{i}(\mathbf{R} / \mathfrak{m})$, a well-known property of cohomological degrees. Finally, in Section 8 , we recast in the context of Buchsbaum-Rim coefficients several questions treated in this paper.

A street view of our results for the convenience of the reader is given in the following table. Let $\mathbf{R}$ be a Noetherian local ring with infinite residue class field and $M$ a finitely generated $\mathbf{R}$-module with $r=\operatorname{dim}_{\mathbf{R}} M \geq 2$. Let $\mathcal{P}(M)$ be the collection of systems $\mathbf{x}=\left\{x_{1}, x_{2}, \ldots, x_{r}\right\}$ of parameters of $M$. In [8] and in this paper, the authors study multiplicity derived numerical functions

$$
\mathbf{f}: \mathcal{P}(M) \longrightarrow \mathbb{N}
$$

on emphasis on the nature of its range

$$
\mathbf{X}_{\mathbf{f}}(M)=\{\mathbf{f}(\mathbf{x}) \mid \mathbf{x} \in \mathcal{P}(M)\} .
$$

For the two functions $e_{1}(\mathbf{x}, M)$ and $\chi_{1}(\mathbf{x} ; M)$, more properly $\mathbf{f}_{1}(\mathbf{x})=-e_{1}(\mathbf{x}, M)$, and $\mathbf{f}_{2}(\mathbf{x})=$ $\chi_{1}(\mathbf{x} ; M)$, respectively, identical assertions about the character of $\mathbf{X}_{\mathbf{f}}(M)$ are expressed in the following grid:

\begin{tabular}{|c|c|c|c|}
\hline $\mathbf{X}_{\mathbf{f}}(M) \subseteq[0, \infty)$ & $M$ & {$[19]$} & {$[26$, Appendice II] } \\
\hline $0 \in \mathbf{X}_{\mathbf{f}}(M)$ & $M$ Cohen-Macaulay & Theorem [3.1 & {$[$ [26, Appendice II] } \\
\hline$\left|\mathbf{X}_{\mathbf{f}}(M)\right|<\infty$ & $M$ generalized Cohen-Macaulay & Theorem $\left[4.5^{*}\right.$ & {$[\underline{6}$} \\
\hline$\left|\mathbf{X}_{\mathbf{f}}(M)\right|=1$ & $M$ Buchsbaum & Theorem $\left[5.4^{*}\right.$ & {$[28]$} \\
\hline$|\{\mathbf{f}(\mathbf{x}) \mid \bar{Q}=\overline{(\mathbf{x})}\}|<\infty$ & $Q$ & Corollary 6.7 & Corollary [7.3 \\
\hline
\end{tabular}

TABLE 1. Properties of a finitely generated module $M$ carried by the values of either function. An adorned reference $[\mathrm{XY}]^{*}$ requires that the module $M$ be unmixed. The third and fourth columns refer to the functions $\mathbf{f}_{1}(\mathbf{x})$ and $\mathbf{f}_{2}(\mathbf{x})$ respectively.

\section{Preliminaries}

Throughout this section let $\mathbf{R}$ be a Noetherian local ring with maximal ideal $\mathfrak{m}$ and let $M$ be a finitely generated $\mathbf{R}$-module. For basic terminology and properties of Noetherian rings and Cohen-Macaulay rings and modules we make use of [3] and [20]. For convenience of exposition we treat briefly the role of hyperplane sections in Hilbert functions and examine unmixed modules. We add further clarifications when we define homological degrees.

Hyperplane sections and Hilbert polynomials. We need rules to compute these coefficients. Typically they involve so called superficial elements or filter regular elements. We keep the terminology of generic hyperplane section, even when dealing with Samuel's multiplicity with respect to an $\mathfrak{m}$-primary ideal $I$ and its Hilbert coefficients $e_{i}(M)=e_{i}(I, M)$. Hopefully this usage will not lead to undue confusion. We say that $h \in I$ is a parameter for $M$, if $\operatorname{dim}_{\mathbf{R}} M / h M<\operatorname{dim}_{\mathbf{R}} M$. 
Let us begin with the following.

Lemma 2.1. Let $(\mathbf{R}, \mathfrak{m})$ be a Noetherian local ring, $I$ an $\mathfrak{m}$-primary ideal of $\mathbf{R}$, and $M$ a finitely generated $\mathbf{R}$-module. Let $h \in I$ and suppose that $\lambda\left(0:_{M} h\right)<\infty$. Then we have the following.

(a) $h$ is a parameter for $M$, if $\operatorname{dim}_{\mathbf{R}} M>0$.

(b) $\lambda\left(0:_{M} h\right) \leq \lambda\left(\mathrm{H}_{\mathfrak{m}}^{0}(M / h M)\right)$.

(c) [24, (1.5)] If $\operatorname{dim}_{\mathbf{R}} M>1$ and $M / h M$ is Cohen-Macaulay, then $M$ is Cohen-Macaulay.

Proof. Suppose that $\operatorname{dim}_{\mathbf{R}} M>0$ and let $\mathfrak{p} \in \operatorname{Supp}_{\mathbf{R}} M$ with $\operatorname{dim} \mathbf{R} / \mathfrak{p}=\operatorname{dim}_{\mathbf{R}} M$. Then

$$
\text { (0) }:_{M_{\mathfrak{p}}} \frac{h}{1}=(0)
$$

since $\mathfrak{p} \neq \mathfrak{m}$. As $\operatorname{dim}_{\mathbf{R}_{\mathfrak{p}}} M_{\mathfrak{p}}=0$, we get $h \notin \mathfrak{p}$. Hence $h$ is a parameter for $M$, if $\operatorname{dim}_{\mathbf{R}} M>0$.

We look at the exact sequence

$0 \rightarrow(0): M h \rightarrow \mathrm{H}_{\mathfrak{m}}^{0}(M) \stackrel{h}{\rightarrow} \mathrm{H}_{\mathfrak{m}}^{0}(M) \stackrel{\varphi}{\rightarrow} \mathrm{H}_{\mathfrak{m}}^{0}(M / h M) \rightarrow \mathrm{H}_{\mathfrak{m}}^{1}(M) \stackrel{h}{\rightarrow} \mathrm{H}_{\mathfrak{m}}^{1}(M) \rightarrow \mathrm{H}_{\mathfrak{m}}^{1}(M / h M) \rightarrow \cdots$

of local cohomology modules derived from the exact sequence

$$
0 \rightarrow(0): M h \rightarrow M \stackrel{h}{\rightarrow} M \rightarrow M / h M \rightarrow 0
$$

of $\mathbf{R}$-modules. We then have

$$
\lambda\left((0):_{M} h\right)=\lambda(\operatorname{Im} \varphi)=\lambda\left(\mathrm{H}_{\mathfrak{m}}^{0}(M / h M)\right)-\lambda\left((0):_{\mathrm{H}_{\mathfrak{m}}^{1}(M)} h\right) \leq \lambda\left(\mathrm{H}_{\mathfrak{m}}^{0}(M / h M)\right) .
$$

Therefore, if $\operatorname{dim}_{\mathbf{R}} M>1$ and $M / h M$ is Cohen-Macaulay, then $h$ is $M$-regular and hence $M$ is Cohen-Macaulay as well.

We will make repeated use of [21, (22.6)] and [19, Section 3]. See also [23] and [24] for a more general version of these results.

Proposition 2.2. Let $(\mathbf{R}, \mathfrak{m})$ be a Noetherian local ring, $I$ an $\mathfrak{m}$-primary ideal of $\mathbf{R}$, and $M$ a finitely generated $\mathbf{R}$-module with $r=\operatorname{dim}_{\mathbf{R}} M>0$. Let $h \in I$ and assume that $h$ is superficial for $M$ with respect to $I$ (in particular $h \in I \backslash \mathfrak{m} I$ ).

(a) The Hilbert coefficients of $M$ and $M / h M$ satisfy

$$
\begin{aligned}
e_{i}(M) & =e_{i}(M / h M) \text { for } 0 \leq i<r-1 \text { and } \\
e_{r-1}(M) & =e_{r-1}(M / h M)+(-1)^{r} \lambda\left(0:_{M} h\right) .
\end{aligned}
$$

(b) Let $0 \rightarrow A \rightarrow B \rightarrow C \rightarrow 0$ be an exact sequence of finitely generated $\mathbf{R}-$ modules. If $t=\operatorname{dim}_{\mathbf{R}} A<s=\operatorname{dim}_{\mathbf{R}} B$, then $e_{i}(B)=e_{i}(C)$ for $0 \leq i<s-t$. In particular, if $t=0$ and $s \geq 2$, then $e_{1}(B)=e_{1}(C)$.

(c) If $M$ is a module of dimension 1 and $I$ is a parameter ideal for $M$, then

$$
e_{1}(M)=-\lambda\left(\mathrm{H}_{\mathfrak{m}}^{0}(M)\right)
$$

(d) If $M$ is a module of dimension 2 and $I$ is a parameter ideal for $M$, then

$$
e_{1}(M)=e_{1}(M / h M)+\lambda\left(0:_{M} h\right)=-\lambda\left(\mathrm{H}_{\mathfrak{m}}^{0}(M / h M)\right)+\lambda\left(0:_{M} h\right)=-\lambda\left((0):_{\mathrm{H}_{\mathfrak{m}}^{1}(M)} h\right) .
$$

Proof. See Proof of Lemma 2.1 for assertion (d).

The following Corollary was previously observed in [19]. By induction on $r=\operatorname{dim}_{\mathbf{R}} M$, it also can be achieved independently, using Proposition 2.2. 
Corollary 2.3. If $M$ is a module of positive dimension and $I$ is a parameter ideal for $M$, then $e_{1}(I, M) \leq 0$.

Unmixed modules. We recall the notion of unmixed local rings and modules and develop a setting to study their Hilbert coefficients.

Definition 2.4. Let $(\mathbf{R}, \mathfrak{m})$ be a Noetherian local ring of dimension $d$. Then we say that $\mathbf{R}$ is unmixed, if $\operatorname{dim} \widehat{\mathbf{R}} / \mathfrak{p}=d$ for every $\mathfrak{p} \in$ Ass $\widehat{\mathbf{R}}$, where $\widehat{\mathbf{R}}$ is the $\mathfrak{m}$-adic completion of $\mathbf{R}$. Similarly, let $M$ be a finitely generated $\mathbf{R}$-module of dimension $r$. Then we say that $M$ is unmixed, if $\operatorname{dim} \widehat{\mathbf{R}} / \mathfrak{p}=r$ for every $\mathfrak{p} \in \operatorname{Ass}_{\widehat{\mathbf{R}}} \widehat{M}$, where $\widehat{M}$ denotes the $\mathfrak{m}$-adic completion of $M$.

Our formulation of unmixedness is the following.

Theorem 2.5. Let $\mathbf{R}$ be a Noetherian local ring and $M$ a finitely generated $\mathbf{R}$-module with $\operatorname{dim}_{\mathbf{R}} M=\operatorname{dim} \mathbf{R}$. Then the following conditions are equivalent:

(i) $M$ is an unmixed $\mathbf{R}-$ module;

(ii) There exists a surjective homomorphism $\mathbf{S} \rightarrow \widehat{\mathbf{R}}$ of rings together with an embedding $\widehat{M} \hookrightarrow \mathbf{S}^{n}$ as an $\mathbf{S}-$ module for some $n>0$, where $\mathbf{S}$ is a Gorenstein local ring with $\operatorname{dim} \mathbf{S}=\operatorname{dim} \mathbf{R}$.

Proof. We have only to prove (i) $\Rightarrow$ (ii). We may assume $\mathbf{R}$ is complete. Thanks to Cohen's structure theorem of complete local rings, we can choose a surjective homomorphism $\mathbf{S} \rightarrow \mathbf{R}$ of rings such that $\mathbf{S}$ is a Gorenstein local $\operatorname{ring}$ with $\operatorname{dim} \mathbf{S}=\operatorname{dim} \mathbf{R}$. Then, because $\operatorname{Asss}_{\mathbf{S}} M \subseteq \operatorname{Ass} \mathbf{S}$ and the $\mathbf{S}_{\mathfrak{p}}$-module $M_{\mathfrak{p}}$ is reflexive for all $\mathfrak{p} \in \operatorname{Ass}_{\mathbf{S}} M$, the canonical map

$$
M \rightarrow \operatorname{Hom}_{\mathbf{S}}\left(\operatorname{Hom}_{\mathbf{S}}(M, \mathbf{S}), \mathbf{S}\right)
$$

is injective, while we get an embedding

$$
\operatorname{Hom}_{\mathbf{S}}\left(\operatorname{Hom}_{\mathbf{S}}(M, \mathbf{S}), \mathbf{S}\right) \hookrightarrow \mathbf{S}^{n}
$$

for some $n>0$, because $\operatorname{Hom}_{\mathbf{S}}(M, \mathbf{S})$ is a finitely generated $\mathbf{S}-$ module. Hence the result.

Corollary 2.6 ([11]). Let $(\mathbf{R}, \mathfrak{m})$ be a Noetherian local ring and $M$ a finitely generated $\mathbf{R}-$ module with $\operatorname{dim}_{\mathbf{R}} M=\operatorname{dim} \mathbf{R} \geq 2$. If $M$ is an unmixed $\mathbf{R}$-module, then $\mathrm{H}_{\mathfrak{m}}^{1}(M)$ is finitely generated.

Proof. We may assume $\mathbf{R}$ is complete. We maintain the notation in Proof of Theorem 2.5] and let $\mathfrak{n}$ denote the maximal ideal of $\mathbf{S}$. Then, applying the functors $\mathrm{H}_{\mathfrak{n}}^{i}(*)$ to the exact sequence

$$
0 \rightarrow M \rightarrow \mathbf{S}^{n} \rightarrow C \rightarrow 0
$$

of $\mathbf{S}$-modules, we get $\mathrm{H}_{\mathfrak{m}}^{1}(M) \cong \mathrm{H}_{\mathfrak{n}}^{0}(C)$, because depthS $\geq 2$. Hence $\mathrm{H}_{\mathfrak{m}}^{1}(M)$ is finitely generated. 


\section{VANishing of $e_{1}(Q, M)$}

Let $\mathbf{R}$ be a Noetherian local ring with maximal ideal $\mathfrak{m}$ and $M$ a finitely generated $\mathbf{R}$-module with $r=\operatorname{dim}_{\mathbf{R}} M$. Recall that a parameter ideal for $M$ is an ideal $Q=\left(x_{1}, x_{2}, \ldots, x_{r}\right) \subseteq \mathfrak{m}$ in $\mathbf{R}$ with $\lambda(M / Q M)<\infty$.

Theorem 3.1. Let $\mathbf{R}$ be a Noetherian local ring and $M$ a finitely generated $\mathbf{R}$-module with $\operatorname{dim}_{\mathbf{R}} M \geq 2$. Suppose that $M$ is unmixed and let $Q$ be a parameter ideal for $M$. Then the following conditions are equivalent:

(i) $M$ is a Cohen-Macaulay $\mathbf{R}-$ module;

(ii) $e_{1}(Q, M)=0$.

Proof. We set $e_{1}(Q)=e_{1}(Q, M)$. It is enough to show that if $M$ is not Cohen-Macaulay, then $e_{1}(Q)<0$. We may assume that $\mathbf{R}$ is complete with an infinite residue field and $\operatorname{dim} \mathbf{R}=$ $\operatorname{dim} M$.

Choose a Gorenstein local ring $(\mathbf{S}, \mathfrak{n})$ and a surjection $\mathbf{S} \rightarrow \mathbf{R}$, with $\operatorname{dim} \mathbf{S}=\operatorname{dim} \mathbf{R}$. If $Q$ is a parameter ideal of $\mathbf{R}$, there exists a parameter ideal $\mathfrak{q}$ of $\mathbf{S}$ such that $\mathfrak{q} \mathbf{R}=Q$ ([9, Lemma 3.1]). Therefore the associated graded module of $Q$ relative to $M$ is isomorphic to the associated graded module of $\mathfrak{q}$ with respect to the $\mathbf{S}$-module $M$ :

$$
\operatorname{gr}_{Q}(M) \simeq \operatorname{gr}_{\mathfrak{q}}(M)
$$

which implies that

$$
e_{1}(Q)=e_{1}(\mathfrak{q}, M)
$$

where $e_{1}(\mathfrak{q}, M)$ denotes the first Hilbert coefficient of $\mathfrak{q}$ with respect to the $\mathbf{S}$-module $M$.

Consider the exact sequence of $\mathbf{S}$-modules obtained from Proposition 2.5.

$$
0 \rightarrow M \rightarrow \mathbf{S}^{n} \rightarrow C \rightarrow 0 .
$$

Let $y$ be a superficial element for $\mathfrak{q}$ with respect to $M$ such that $y$ is part of a minimal generating set of $\mathfrak{q}$. We may assume that $y$ is a nonzero divisor on $M$. By tensoring the exact sequence of $\mathbf{S}-$ modules with $\mathbf{S} /(y)$, we get

$$
0 \rightarrow T=\operatorname{Tor}_{1}^{\mathbf{S}}(\mathbf{S} /(y), C) \rightarrow M / y M \stackrel{\zeta}{\rightarrow} \mathbf{S}^{n} / y \mathbf{S}^{n} \rightarrow C / y C \rightarrow 0 .
$$

Let $M^{\prime}=M / y M$ and $N=\operatorname{Im}(\zeta)$ and consider the short exact sequence:

$$
0 \rightarrow T \rightarrow M^{\prime} \rightarrow N \rightarrow 0 .
$$

Then either $T=0$ or $T$ has finite length $\lambda(T)<\infty$. Note that $N$ is an unmixed $\mathbf{S} /(y)$-module.

We use induction on $d=\operatorname{dim} M$ to show that if $M$ is not Cohen-Macaulay, then $e_{1}(\mathfrak{q}, M)<0$.

Let $d=2$ and $\mathfrak{q}=(y, z)$. Then $T \neq 0$ so that $\lambda(T)<\infty$. Applying the Snake Lemma to

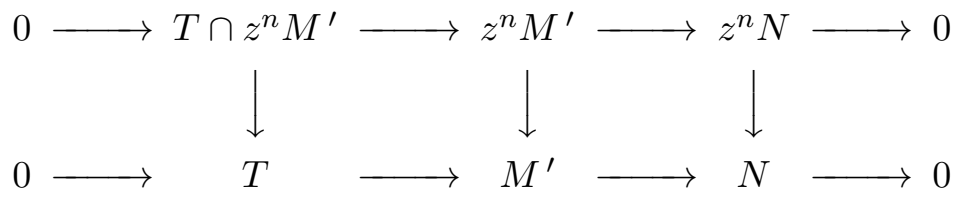

we get, for sufficiently large $n$,

$$
\lambda\left(M^{\prime} / z^{n} M^{\prime}\right)=\lambda(T)+\lambda\left(N / z^{n} N\right) .
$$


Computing the Hilbert polynomials, we have

$$
e_{1}(\mathfrak{q}, M)=e_{1}(\mathfrak{q} /(y), M / y M)=-\lambda(T)<0 .
$$

Now suppose that $d \geq 3$. From the exact sequence

$$
0 \rightarrow T \rightarrow M^{\prime}=M / y M \rightarrow N \rightarrow 0,
$$

we have

$$
e_{1}(\mathfrak{q}, M)=e_{1}(\mathfrak{q} /(y), M / y M)=e_{1}(\mathfrak{q} /(y), N) .
$$

By an induction argument, it is enough to show that $N$ is not Cohen-Macaulay since $\operatorname{dim}(\mathbf{S} /(y))=$ $d-1$.

Suppose that $N$ is Cohen-Macaulay. Let $\mathfrak{n}$ be the maximal ideal of $\mathbf{S} / y \mathbf{S}$. From the exact sequence

$$
0 \rightarrow T \rightarrow M^{\prime}=M / y M \rightarrow N \rightarrow 0,
$$

we obtain the long exact sequence:

$$
0 \rightarrow \mathrm{H}_{\mathfrak{n}}^{0}(T) \rightarrow \mathrm{H}_{\mathfrak{n}}^{0}\left(M^{\prime}\right) \rightarrow \mathrm{H}_{\mathfrak{n}}^{0}(N) \rightarrow \mathrm{H}_{\mathfrak{n}}^{1}(T) \rightarrow \mathrm{H}_{\mathfrak{n}}^{1}\left(M^{\prime}\right) \rightarrow \mathrm{H}_{\mathfrak{n}}^{1}(N)
$$

By the assumption that $N$ is Cohen-Macaulay of dimension $d-1 \geq 2$ and the fact that $T$ is a torsion module, we get

$$
0 \rightarrow T \simeq \mathrm{H}_{\mathfrak{n}}^{0}\left(M^{\prime}\right) \rightarrow 0 \rightarrow 0 \rightarrow \mathrm{H}_{\mathfrak{n}}^{1}\left(M^{\prime}\right) \rightarrow 0 .
$$

From the exact sequence

$$
0 \rightarrow M \stackrel{\cdot y}{\rightarrow} M \rightarrow M^{\prime}=M / y M \rightarrow 0
$$

we obtain the following exact sequence:

$$
0 \rightarrow T \simeq \mathrm{H}_{\mathfrak{n}}^{0}\left(M^{\prime}\right) \rightarrow \mathrm{H}_{\mathfrak{n}}^{1}(M) \stackrel{\cdot y}{\rightarrow} \mathrm{H}_{\mathfrak{n}}^{1}(M) \rightarrow \mathrm{H}_{\mathfrak{n}}^{1}\left(M^{\prime}\right)=0 .
$$

Since $\mathrm{H}_{\mathfrak{n}}^{1}(M)$ is finitely generated by Corollary 2.6 and $\mathrm{H}_{\mathfrak{n}}^{1}(M)=y \mathrm{H}_{\mathfrak{n}}^{1}(M)$, we have $\mathrm{H}_{\mathfrak{n}}^{1}(M)=0$. This means that $T=0$. Therefore

$$
0 \rightarrow T=0 \rightarrow M / y M \simeq N \rightarrow 0 .
$$

Since $N$ is Cohen-Macaulay, $M / y M$ is Cohen-Macaulay. Since $y$ is regular on $M, M$ is Cohen-Macaulay, which is a contradiction.

Example 3.2. ([32]). Let $M=\mathbf{R}=k[[x, y, z]] /(z(x, y, z))$. Then $H_{\mathfrak{m}}^{0}(\mathbf{R})=(z)$, and $\mathbf{S}=$ $\mathbf{R} / H_{\mathfrak{m}}^{0}(\mathbf{R}) \simeq k[[x, y]]$ is Cohen-Macaulay. If $Q$ is a parameter ideal of $\mathbf{R}$, then $e_{1}(Q, \mathbf{R})=$ $e_{1}(Q \mathbf{S}, \mathbf{S})=0$. Hence $e_{1}(Q, \mathbf{R})=0$, but $\mathbf{R}$ is not Cohen-Macaulay. Therefore the unmixdness condition is necessary Theorem 3.1 .

Let us list some consequences of Theorem 3.1. Let $\mathbf{R}$ be a Noetherian local $\operatorname{ring}$ and $M$ a finitely generated $\mathbf{R}$-module. We put

$$
\operatorname{Assh}_{\mathbf{R}} M=\left\{\mathfrak{p} \in \operatorname{Ass}_{\mathbf{R}} M \mid \operatorname{dim} \mathbf{R} / \mathfrak{p}=\operatorname{dim}_{\mathbf{R}} M\right\} .
$$


Let $\left(0_{M}\right)=\bigcap_{\mathfrak{p} \in \operatorname{Ass}_{\mathbf{R}} M} M(\mathfrak{p})$ be a primary decomposition of $0_{M}$ in $M$, where $M(\mathfrak{p})$ is a $\mathfrak{p}-$ primary submodule of $M$ for each $\mathfrak{p} \in \operatorname{Ass}_{\mathbf{R}} M$. We put

$$
\mathrm{U}_{M}(0)=\bigcap_{\mathfrak{p} \in \operatorname{Assh}_{\mathbf{R}} M} M(\mathfrak{p})
$$

and call it the unmixed component of $M$. We then have the following.

Lemma 3.3. Let $\mathbf{R}$ be a Noetherian local ring and $M$ a finitely generated $\mathbf{R}$-module with $r=\operatorname{dim}_{\mathbf{R}} M>0$. Let $Q$ be a parameter ideal for $M$. Let $U=\mathrm{U}_{M}(0)$ and suppose that $U \neq(0)$. We put $N=M / U$. Then the following assertions hold.

(a) $\operatorname{dim}_{\mathbf{R}} U<\operatorname{dim}_{\mathbf{R}} M$.

(b)

$$
e_{1}(Q, M)= \begin{cases}e_{1}(Q, N) & \text { if } \operatorname{dim}_{\mathbf{R}} U \leq r-2, \\ e_{1}(Q, N)-s_{0} & \text { if } \operatorname{dim}_{\mathbf{R}} U=r-1,\end{cases}
$$

where $s_{0} \geq 1$ denotes the multiplicity of the graded $\operatorname{gr}_{Q}(\mathbf{R})$-module $\bigoplus_{n \geq 0} U /\left(Q^{n+1} M \cap U\right)$.

(c) $e_{1}(Q, M) \leq e_{1}(Q, N)$ and the equality $e_{1}(Q, M)=e_{1}(Q, N)$ holds if and only if $\operatorname{dim}_{\mathbf{R}} U \leq r-2$.

Proof. (a) This is clear, since $\mathrm{U}_{\mathfrak{p}}=(0)$ for all $\mathfrak{p} \in \operatorname{Assh}_{\mathbf{R}} M$.

(b) We write

$$
\lambda_{\mathbf{R}}\left(U /\left(Q^{n+1} M \cap U\right)\right)=s_{0}\left(\begin{array}{c}
n+t \\
t
\end{array}\right)-s_{1}\left(\begin{array}{c}
n+t-1 \\
t-1
\end{array}\right)+\cdots+(-1)^{t} s_{t}
$$

for $n \gg 0$ with integers $\left\{s_{i}\right\}_{0 \leq i \leq t}$, where $t=\operatorname{dim}_{\mathbf{R}} U$. Then the claim follows from the exact sequence $0 \rightarrow U \rightarrow M \rightarrow N \rightarrow 0$ of $\mathbf{R}$-modules, which gives

$$
\lambda_{\mathbf{R}}\left(M / Q^{n+1} M\right)=\lambda_{\mathbf{R}}\left(N / Q^{n+1} N\right)+\lambda_{\mathbf{R}}\left(U /\left(Q^{n+1} M \cap U\right)\right), \quad \forall n \geq 0 .
$$

(c) This follows from (b) and the fact that $s_{0} \geq 1$.

Theorem 3.4. Let $\mathbf{R}$ be a Noetherian local ring and $M$ a finitely generated $\mathbf{R}$-module with $r=\operatorname{dim}_{\mathbf{R}} M \geq 2$. Suppose that $\mathbf{R}$ is a homomorphic image of a Cohen-Macaulay ring. Let $U=\mathrm{U}_{M}(0)$ and let $Q$ be a parameter ideal for $M$. Then the following conditions are equivalent:

(i) $e_{1}(Q, M)=0$;

(ii) $M / U$ is a Cohen-Macaulay $\mathbf{R}$-module and $\operatorname{dim}_{\mathbf{R}} U \leq r-2$.

Proof. It is enough to prove (i) $\Rightarrow$ (ii). If $\operatorname{dim}_{\mathbf{R}} U=r-1$, then by (i) and Lemma [3.3-(b), we obtain $0 \geq e_{1}(Q, M / U)=s_{0} \geq 1$, which is a contradiction. Hence $\operatorname{dim}_{\mathbf{R}} U \leq r-2$. This means that $0=e_{1}(Q, M)=e_{1}(Q, M / U)$. By Theorem 3.1, $M / U$ is Cohen-Macaulay.

The implication (i) $\Rightarrow$ (ii) in Theorem 3.4 is not true in general without the assumption that $\mathbf{R}$ is a homomorphic image of a Cohen-Macaulay ring. See [8, Remark 2] for an example. The following corollary gives a characterization of Cohen-Macaulayness. 
Corollary 3.5. Let $\mathbf{R}$ be a Noetherian local ring, $M$ a finitely generated $\mathbf{R}$-module with $r=\operatorname{dim}_{\mathbf{R}} M>0$, and $Q$ a parameter ideal for $M$. Let $1 \leq k \leq r$ be an integer and assume that $e_{i}(Q, M)=0$ for all $1 \leq i \leq k$. Then

$$
\operatorname{dim}_{\widehat{\mathbf{R}}} \mathrm{U}_{\widehat{M}}(0) \leq r-(k+1) \text { and } \mathrm{H}_{\mathfrak{m}}^{r-j}(M)=(0)
$$

for all $1 \leq j \leq k$. In particular, if $k=r$, then $M$ is a Cohen-Macaulay $\mathbf{R}$-module.

Proof. We may assume that $\mathbf{R}$ is complete. We put $U=\mathrm{U}_{M}(0)$ and $N=M / U$. Then $e_{0}(Q, M)=e_{0}(Q, N)$, since $\operatorname{dim}_{\mathbf{R}} U<r$. Therefore, by Theorem $3.4 N$ is a Cohen-Macaulay $\mathbf{R}$-module, so that we have exact sequences

$$
0 \rightarrow U / Q^{n+1} U \rightarrow M / Q^{n+1} M \rightarrow N / Q^{n+1} N \rightarrow 0
$$

of $\mathbf{R}$-modules for all $n \geq 0$. Hence, computing Hilbert polynomials, we get $\operatorname{dim}_{\mathbf{R}} U \leq r-(k+1)$. Let $1 \leq j \leq k$. Then $\mathrm{H}_{\mathfrak{m}}^{r-j}(U)=(0)$, since $\operatorname{dim}_{\mathbf{R}} U<r-j$, while $\mathrm{H}_{\mathfrak{m}}^{r-j}(N)=(0)$, as $N$ is a Cohen-Macaulay $\mathbf{R}$-module with $\operatorname{dim}_{\mathbf{R}} N=r$. Thus $\mathrm{H}_{\mathfrak{m}}^{r-j}(M)=(0)$ as claimed.

Let $\mathbf{R}$ be a Noetherian local $\operatorname{ring}$ and $M$ a finitely generated $\mathbf{R}$-module. In $[8$, the authors examined the rings with $e_{1}(Q, R)$ vanishing. Here we briefly extend this theory to modules. Let us begin with the definition.

Definition 3.6. A finitely generated $\mathbf{R}$-module $M$ is called a Vasconcelos modul 1 , if either $\operatorname{dim}_{\mathbf{R}} M=0$, or $\operatorname{dim}_{\mathbf{R}} M>0$ and $e_{1}(Q, M)=0$ for some parameter ideal $Q$ for $M$.

Every Cohen-Macaulay module is by definition Vasconcelos. Here is a basic characterization. We omit the proof, since it is similar to those in the ring case.

Theorem 3.7. Let $(\mathbf{R}, \mathfrak{m})$ be a Noetherian local ring and $M$ a finitely generated $\mathbf{R}$-module with $r=\operatorname{dim}_{\mathbf{R}} M \geq 2$. Let $U=\mathrm{U}_{\widehat{M}}(0)$ be the unmixed component of $(0)$ in the $\mathfrak{m}$-adic completion $\widehat{M}$ of $M$. Then the following conditions are equivalent:

(i) $M$ is a Vasconcelos $\mathbf{R}$-module;

(ii) $e_{1}(Q, M)=0$ for every parameter ideal $Q$ for $M$;

(iii) $\widehat{M} / \mathrm{U}_{\widehat{M}}(0)$ is a Cohen-Macaulay $\widehat{\mathbf{R}}$-module and $\operatorname{dim}_{\widehat{\mathbf{R}}} \mathrm{U}_{\widehat{M}}(0) \leq r-2$;

(iv) There exists a proper $\widehat{\mathbf{R}}$-submodule $L$ of $\widehat{M}$ such that $\widehat{M} / L$ is a Cohen-Macaulay $\widehat{\mathbf{R}}$ module with $\operatorname{dim}_{\widehat{\mathbf{R}}} L \leq r-2$.

When this is the case, $\widehat{M}$ is a Vasconcelos $\widehat{\mathbf{R}}-$ module and $\mathrm{H}_{\mathfrak{m}}^{r-1}(M)=(0)$.

Remark 3.8. Several properties of Vasconcelos rings such as [8, 3.5, 3.8, 3.9, 3.10, 3.11, 3.12, $3.13,3.15,3.16,3.17]$ can be all extended to Vasconcelos modules.

\section{Generalized Cohen-Macaulayness of modules with $\Lambda(M)$ finite}

Let $\mathbf{R}$ be a Noetherian local ring with maximal ideal $\mathfrak{m}$ and $M$ a finitely generated $\mathbf{R}$-module with $r=\operatorname{dim}_{\mathbf{R}} M>0$. In this section we study the problem of when the set

$$
\Lambda(M)=\left\{e_{1}(Q, M) \mid Q \text { is a parameter ideal for } M\right\}
$$

is finite. Part of the motivation comes from the fact that generalized Cohen-Macaulay modules have this property. Recall that $M$ is said to be generalized Cohen-Macaulay, if all the

\footnotetext{
${ }^{1}$ The terminology is due to the other five authors.
} 
local cohomology modules $\left\{\mathrm{H}_{\mathfrak{m}}^{i}(M)\right\}_{0 \leq i<r}$ are finitely generated (see [6] where these modules originated).

Assume that $M$ is a generalized Cohen-Macaulay $\mathbf{R}$-module with $r=\operatorname{dim}_{\mathbf{R}} M \geq 2$ and put

$$
s=\sum_{i=1}^{r-1}\left(\begin{array}{l}
r-2 \\
i-1
\end{array}\right) h^{i}(M),
$$

where $h^{i}(M)=\lambda_{\mathbf{R}}\left(\mathrm{H}_{\mathfrak{m}}^{i}(M)\right)$ for each $i \in \mathbb{Z}$. If $Q$ is a parameter ideal for $M$, by the proof of [12, Lemma 2.4] we have that $e_{1}(Q, M) \geq-s$. Since $e_{1}(Q, M) \leq 0$ by Corollary 2.3, it follows that $\Lambda(M)$ is a finite set.

Let us establish here that if $M$ is unmixed and $\Lambda(M)$ is finite, then $M$ is indeed a generalized Cohen-Macaulay R-module (Proposition 4.2).

Assume now that $\mathbf{R}$ is a homomorphic image of a Gorenstein local ring and that $\operatorname{Ass}_{\mathbf{R}} M=$ $\operatorname{Assh}_{\mathbf{R}} M$. Then $\mathbf{R}$ contains a system $x_{1}, x_{2}, \ldots, x_{r}$ of parameters of $M$ which forms a strong $d$-sequence for $M$, that is, the sequence $x_{1}^{n_{1}}, x_{2}^{n_{2}}, \ldots, x_{r}^{n_{r}}$ is a $d$-sequence for $M$ for all integers $n_{1}, n_{2}, \ldots, n_{r} \geq 1$ (see [5, Theorem 2.6] or [17, Theorem 4.2] for the existence of such systems of parameters). For each integer $q \geq 1$ let $\Lambda_{q}(M)$ be the set of values $e_{1}(Q, M)$, where $Q$ runs over the parameter ideals for $M$ such that $Q \subseteq \mathfrak{m}^{q}$ and $Q=\left(x_{1}, x_{2}, \ldots, x_{r}\right)$ with $x_{1}, x_{2}, \ldots, x_{r}$ a $d$-sequence for $M$. We then have $\Lambda_{q}(M) \neq \emptyset, \Lambda_{q+1}(M) \subseteq \Lambda_{q}(M)$ for all $q \geq 1$, and $\alpha \leq 0$ for every $\alpha \in \Lambda_{q}(M)$ (Corollary 2.3).

The following result plays a key role in our argument. The proof which we present here is based on Theorem 2.5 and slightly different from that of the ring case.

Lemma 4.1. Let $(\mathbf{R}, \mathfrak{m})$ be a Noetherian local ring and assume that $\mathbf{R}$ is a homomorphic image of a Gorenstein ring. Let $M$ be a finitely generated $\mathbf{R}$-module with $r=\operatorname{dim}_{\mathbf{R}} M \geq 2$ and $\operatorname{Ass}_{\mathbf{R}} M=\operatorname{Assh}_{\mathbf{R}} M$. Assume that $\Lambda_{q}(M)$ is a finite set for some integer $q \geq 1$ and put $\ell=-\min \Lambda_{q}(M)$. Then $\mathfrak{m}^{\ell} \mathrm{H}_{\mathfrak{m}}^{i}(M)=(0)$ for all $i \neq r$ and hence all the local cohomology modules $\left\{\mathrm{H}_{\mathfrak{m}}^{i}(M)\right\}_{0 \leq i<r}$ are finitely generated.

Proof. Passing to the $\operatorname{ring} \mathbf{R} /\left[(0):_{\mathbf{R}} M\right]$, we may assume that $\mathbf{R}$ is a Gorenstein ring with $\operatorname{dim} \mathbf{R}=\operatorname{dim}_{\mathbf{R}} M=r$. Enlarging the residue class field $\mathbf{R} / \mathfrak{m}$ of $\mathbf{R}$ if necessary, we may assume the field $\mathbf{R} / \mathfrak{m}$ is infinite. By Corollary $2.6 \mathrm{H}_{\mathfrak{m}}^{1}(M)$ is finitely generated, since $M$ is unmixed.

Suppose that $r=2$. We put $\ell^{\prime}=\lambda\left(\mathrm{H}_{\mathfrak{m}}^{1}(M)\right)$. Let $Q=(x, y) \subseteq \mathfrak{m}^{q}$ be a system of parameters for $M$ such that $Q \mathrm{H}_{\mathfrak{m}}^{1}(M)=(0)$ and $x, y$ is a $d$-sequence for $M$. Then $x$ is superficial for $M$ with respect to $Q$. Hence by Proposition $2.2(\mathrm{~d})$ we get $e_{1}(Q, M)=-\lambda\left(\mathrm{H}_{\mathfrak{m}}^{1}(M)\right)=-\ell^{\prime}$. Thus $\ell \geq \ell^{\prime}$, as $-\ell^{\prime}=e_{1}(Q, M) \in \Lambda_{q}(M)$. Hence $\mathfrak{m}^{\ell} \mathrm{H}_{\mathfrak{m}}^{1}(M)=(0)$, because $\mathfrak{m}^{\ell^{\prime}} \mathrm{H}_{\mathfrak{m}}^{1}(M)=(0)$.

Suppose that $r \geq 3$ and that our assertion holds true for $r-1$. We have an exact sequence

$$
(\sharp) \quad 0 \rightarrow M \rightarrow \mathbf{R}^{n} \rightarrow C \rightarrow 0
$$

of $\mathbf{R}$-modules by Theorem 2.5 . Choose an $\mathbf{R}$-regular element $x \in \mathbf{R}$ so that $x$ is superficial both for $M$ and $C$ with respect to $\mathfrak{m}$. Let us fix an integer $m \geq 1$. We put $y=x^{m}, N=M / y M$, and look at the exact sequence

$$
0 \rightarrow(0):_{C} y \rightarrow N \stackrel{\varphi}{\rightarrow}(\mathbf{R} / y \mathbf{R})^{n} \rightarrow C / y C \rightarrow 0
$$

of $\mathbf{R}$-modules obtained by sequence $(\sharp)$. Let $L=\operatorname{Im} \varphi$. Then $\operatorname{dim}_{\mathbf{R}} L=r-1, \operatorname{Ass}_{\mathbf{R}} L=$ $\operatorname{Assh}_{\mathbf{R}} L$, and $\mathrm{H}_{\mathfrak{m}}^{0}(N) \cong(0):_{C} y$, because $L$ is an $\mathbf{R}$-submodule of $(\mathbf{R} / y \mathbf{R})^{n}$ and $\lambda\left((0):_{C} y\right)<$ $\infty$. Hence $L \cong N / \mathrm{H}_{\mathfrak{m}}^{0}(N)$. 
Let $q^{\prime} \geq q$ be an integer such that $\mathfrak{m}^{q^{\prime}} N \cap \mathrm{H}_{\mathfrak{m}}^{0}(N)=(0)$. Let $y_{2}, y_{3}, \ldots, y_{r} \in \mathfrak{m}^{q^{\prime}}$ be a system of parameters for $L$ and assume that $y_{2}, y_{3}, \ldots, y_{r}$ is a $d$-sequence for $L$. Then, since $\left(y_{2}, y_{3}, \ldots, y_{r}\right) N \cap \mathrm{H}_{\mathfrak{m}}^{0}(N)=(0)$, we get $y_{2}, y_{3}, \ldots, y_{r}$ forms a $d$-sequence for $N$ also. Therefore, since $y$ is $M$-regular, the sequence $y_{1}=y, y_{2}, \ldots, y_{r}$ forms a $d$-sequence for $M$, whence $y_{1}$ is superficial for $M$ with respect to $Q=\left(y_{1}, y_{2}, \ldots, y_{r}\right)$. Consequently

$$
e_{1}\left(\left(y_{2}, y_{3}, \ldots, y_{r}\right), L\right)=e_{1}\left(\left(y_{2}, y_{3}, \ldots, y_{r}\right), N\right)=e_{1}(Q, M) \in \Lambda_{q}(M)
$$

so that $\Lambda_{q^{\prime}}(L) \subseteq \Lambda_{q}(M)$. Hence, because the set $\Lambda_{q^{\prime}}(L)$ is finite, the hypothesis of induction on $r$ yields that $\mathfrak{m}^{\ell^{\prime \prime}} \mathrm{H}_{\mathfrak{m}}^{i}(L)=(0)$ for all $i \neq r-1$, where $\ell^{\prime \prime}=-\min \Lambda_{q^{\prime}}(L)$. Thus, because $\ell^{\prime \prime} \leq \ell, \mathfrak{m}^{\ell} \mathrm{H}_{\mathfrak{m}}^{i}(L)=(0)$ for all $i \neq r-1$. Hence $\mathfrak{m}^{\ell} \mathrm{H}_{\mathfrak{m}}^{i}(N)=(0)$ for all $1 \leq i<r-1$, because $\mathrm{H}_{\mathfrak{m}}^{i}(N) \cong \mathrm{H}_{\mathfrak{m}}^{i}(L)$ for $i \geq 1$.

Look now at the exact sequence

$$
(\sharp \sharp) \quad \cdots \rightarrow \mathrm{H}_{\mathfrak{m}}^{1}(M) \stackrel{x^{m}}{\rightarrow} \mathrm{H}_{\mathfrak{m}}^{1}(M) \rightarrow \mathrm{H}_{\mathfrak{m}}^{1}(N) \rightarrow \cdots \rightarrow \mathrm{H}_{\mathfrak{m}}^{i}(N) \rightarrow \mathrm{H}_{\mathfrak{m}}^{i+1}(M) \stackrel{x^{m}}{\rightarrow} \mathrm{H}_{\mathfrak{m}}^{i+1}(M) \rightarrow \cdots
$$

of local cohomology modules. We then have

$$
\mathfrak{m}^{\ell}\left[(0):_{\mathrm{H}_{\mathfrak{m}}^{i+1}(M)} x^{m}\right]=(0)
$$

for all integers $1 \leq i \leq r-2$ and $m \geq 1$, since $\mathfrak{m}^{\ell} \mathrm{H}_{\mathfrak{m}}^{i}(N)=(0)$ for all $1 \leq i \leq r-2$. Thus $\mathfrak{m}^{\ell} \mathrm{H}_{\mathfrak{m}}^{i+1}(M)=(0)$, because

$$
\mathrm{H}_{\mathfrak{m}}^{i+1}(M)=\bigcup_{m \geq 1}\left[(0):_{\mathrm{H}_{\mathfrak{m}}^{i+1}(M)} \mathfrak{m}^{m}\right]
$$

On the other hand, from sequence $(\sharp \sharp)$ we get the embedding $\mathrm{H}_{\mathfrak{m}}^{1}(M) \subseteq \mathrm{H}_{\mathfrak{m}}^{1}(N)$, choosing the integer $m \geq 1$ so that $x^{m} \mathrm{H}_{\mathfrak{m}}^{1}(M)=(0)$. Hence $\mathfrak{m}^{\ell} \mathrm{H}_{\mathfrak{m}}^{1}(M)=(0)$, which completes the proof of Lemma 4.1 .

Since $\Lambda(M)=\Lambda(\widehat{M})$, passing to the completion $\widehat{M}$ of $M$ and applying Lemma 4.1, we readily get the following.

Proposition 4.2. Let $(\mathbf{R}, \mathfrak{m})$ be a Noetherian local ring and $M$ a finitely generated unmixed $\mathbf{R}$-module with $r=\operatorname{dim}_{\mathbf{R}} M \geq 2$. Assume that $\Lambda(M)$ is a finite set and put $\ell=-\min \Lambda(M)$. Then $\mathfrak{m}^{\ell} \mathrm{H}_{\mathfrak{m}}^{i}(M)=(0)$ for every $i \neq r$, so that $M$ is a generalized Cohen-Macaulay $\mathbf{R}-$ module.

We conclude this section with a characterization of $\mathbf{R}$-modules for which $\Lambda(M)$ is finite.

Let us note the following with a brief proof.

Lemma 4.3. Let $\mathbf{R}$ be a Noetherian local ring and $M$ a finitely generated $\mathbf{R}$-module with $r=\operatorname{dim}_{\mathbf{R}} M \geq 2$. Assume that there exists an integer $t \geq 0$ such that $e_{1}(Q, M) \geq-t$ for every parameter ideal $Q$ for $M$. Then $\operatorname{dim}_{\mathbf{R}} \mathrm{U}_{M}(0) \leq r-2$.

Proof. Let $U=\mathrm{U}_{M}(0)$ and $N=M / U$. Assume that $\operatorname{dim}_{\mathbf{R}} U=r-1$. Choose a system $x_{1}, x_{2}, \ldots, x_{r}$ of parameters of $M$ such that $x_{r} U=(0)$. Let $\ell>t$ be an integer and put $Q=\left(x_{1}^{\ell}, x_{2}, \ldots, x_{r}\right)$. Then we get exact sequences

$$
0 \rightarrow U /\left(Q^{n+1} M \cap U\right) \rightarrow M / Q^{n+1} M \rightarrow N / Q^{n+1} N \rightarrow 0
$$

of $\mathbf{R}$-modules for all $n \geq 0$. Let us take an integer $k \geq 0$ so that

$$
Q^{n} M \cap U=Q^{n-k}\left(Q^{k} M \cap U\right)
$$


for $n \geq k$ and consider $U^{\prime}=Q^{k} M \cap U$. Let $\mathfrak{q}=\left(x_{1}^{\ell}, x_{2}, \ldots, x_{r-1}\right)$. We then have

$$
Q^{n-k} U^{\prime}=\mathfrak{q}^{n-k} U^{\prime}
$$

as $x_{r} U=(0)$. Hence for all $n \geq k$

$$
\lambda_{\mathbf{R}}\left(M / Q^{n+1} M\right)=\lambda_{\mathbf{R}}\left(N / Q^{n+1} N\right)+\lambda_{\mathbf{R}}\left(U^{\prime} / \mathfrak{q}^{n-k+1} U^{\prime}\right)+\lambda_{\mathbf{R}}\left(U / U^{\prime}\right),
$$

which yields $-t \leq e_{1}(Q, M)=e_{1}(Q, N)-e_{0}\left(\mathfrak{q}, U^{\prime}\right)$. Hence

$$
-t \leq e_{1}(Q, M)=e_{1}(Q, N)-e_{0}(\mathfrak{q}, U)
$$

because $e_{0}(\mathfrak{q}, U)=e_{0}\left(\mathfrak{q}, U^{\prime}\right)$ (remember that $\left.\lambda\left(U / U^{\prime}\right)<\infty\right)$. Therefore, since $e_{1}(Q, N) \leq 0$ by Corollary 2.3, we get

$$
\ell \leq \ell e_{0}\left(\left(x_{1}, x_{2}, \ldots, x_{r-1}\right), U\right)=e_{0}(\mathfrak{q}, U) \leq e_{1}(Q, N)+t \leq t,
$$

which is impossible. Thus $\operatorname{dim}_{\mathbf{R}} U \leq r-2$.

Remark 4.4. Let $\mathbf{R}$ be a Noetherian local ring and $M$ a finitely generated $\mathbf{R}$-module with $r=\operatorname{dim}_{\mathbf{R}} M \geq 2$. Assume that $\operatorname{dim}_{\mathbf{R}} \mathrm{U}_{M}(0) \leq r-2$. Let $\mathfrak{q}$ be a parameter ideal for $N=M / \mathrm{U}_{M}(0)$. Then one can find a parameter ideal $Q$ for $M$ with $Q N=\mathfrak{q} N$, so that $e_{1}(\mathfrak{q}, N)=e_{1}(\mathfrak{q}, M)$ by Lemma 3.3. Hence $\Lambda(M)=\Lambda(N)$.

The goal of this section is the following.

Theorem 4.5. Let $(\mathbf{R}, \mathfrak{m})$ be a Noetherian local ring and $M$ a finitely generated $\mathbf{R}$-module with $r=\operatorname{dim}_{\mathbf{R}} M \geq 2$. Let $U=\mathrm{U}_{\widehat{M}}(0)$ denote the unmixed component of (0) in the $\mathfrak{m}$-adic completion $\widehat{M}$ of $M$. Then the following conditions are equivalent:

(i) $\Lambda(M)$ is a finite set;

(ii) $\widehat{M} / U$ is a generalized Cohen-Macaulay $\widehat{\mathbf{R}}-$ module and $\operatorname{dim}_{\widehat{\mathbf{R}}} U \leq r-2$.

When this is the case, one has the estimation

$$
0 \geq e_{1}(Q, M) \geq-\sum_{i=1}^{r-1}\left(\begin{array}{c}
r-2 \\
i-1
\end{array}\right) h^{i}(\widehat{M} / U)
$$

for every parameter ideal $Q$ for $M$.

Proof. We may assume that $\mathbf{R}$ is complete.

(i) $\Rightarrow$ (ii) Since the set $\Lambda(M)$ is finite, by Proposition 4.3 we get $\operatorname{dim}_{\mathbf{R}} U \leq r-2$. By Remark 4.4 the set $\Lambda(M / U)$ is finite, so that $M / U$ is a generalized Cohen-Macaulay $\mathbf{R}$-module by Proposition 4.2 .

(ii) $\Rightarrow$ (i) By [12, Lemma 2.4] the set $\Lambda(M / U)$ is finite and hence the set $\Lambda(M)$ is also finite by Lemma 3.3 .

See [12, Lemma 2.4] for the last assertion. 
5. Buchsbaumness of modules possessing COnStant first Hilbert COefficients of PARAMETERS

Let $\mathbf{R}$ be a Noetherian local ring with maximal ideal $\mathfrak{m}$ and $M$ a finitely generated $\mathbf{R}$-module with $r=\operatorname{dim}_{\mathbf{R}} M>0$. In this section we study the problem of when $e_{1}(Q, M)$ is independent of the choice of parameter ideals $Q$ for $M$. Part of the motivation comes from the fact that Buchsbaum modules have this property. We establish here that if $e_{1}(Q, M)$ is constant and $M$ is unmixed, then $M$ is indeed a Buchsbaum $\mathbf{R}$-module (Theorem [5.4). See [13] for the ring case.

First of all let us recall some definitions. A system $x_{1}, x_{2}, \ldots, x_{r}$ of parameters of $M$ is said to be standard, if it forms a $d^{+}$-sequence for $M$, that is, $x_{1}, x_{2}, \ldots, x_{r}$ forms a strong $d$-sequence for $M$ in any order. Remember that $M$ possesses a standard system of parameters if and only if $M$ is a generalized Cohen-Macaulay $\mathbf{R}$-module ([29]).

Let $Q$ be a parameter ideal for $M$. Then we say that $Q$ is standard, if it is generated by a standard system of parameters of $M$. Remember that $Q$ is standard if and only if the equality

$$
\lambda_{\mathbf{R}}(M / Q M)-e_{0}(Q, M)=\sum_{i=0}^{r-1}\left(\begin{array}{c}
r-1 \\
i
\end{array}\right) h^{i}(M):=\mathbb{I}(M)
$$

holds ([29, Theorem 2.1]). It is known that every system of parameters of $M$ contained in a standard parameter ideal for $M$ is standard ([29]).

Suppose that $M$ is a generalized Cohen-Macaulay $\mathbf{R}$-module with $r=\operatorname{dim}_{\mathbf{R}} M \geq 2$ and $s=\sum_{i=1}^{r-1}\left(\begin{array}{c}r-2 \\ i-1\end{array}\right) h^{i}(M)$. If $Q$ is a parameter ideal for $M$, then by [12, Lemma 2.4] we get $e_{1}(Q, M) \geq-s$, where the equality holds if $Q$ is standard ([25, Korollar 3.2]).

We say that our $\mathbf{R}$-module $M$ is Buchsbaum, if every parameter ideal for $M$ is standard. Hence, if $M$ is a Buchsbaum $\mathbf{R}$-module with $r=\operatorname{dim}_{\mathbf{R}} M \geq 2$, then $M$ is a generalized Cohen-Macaulay $\mathbf{R}$-module with

$$
e_{1}(Q, M)=-\sum_{i=1}^{r-1}\left(\begin{array}{l}
r-2 \\
i-1
\end{array}\right) h^{i}(M)
$$

for every parameter ideal $Q$. See [28] for a detailed theory of Buchsbaum rings and modules.

We begin with the following two results, whose proofs are similar to those in the ring case (see [8, Lemma 4.5] and [13, Proposition 2.3]).

Lemma 5.1. Let $(\mathbf{R}, \mathfrak{m})$ be a Noetherian local ring and $M$ a generalized Cohen-Macaulay $\mathbf{R}$-module with $r=\operatorname{dim}_{\mathbf{R}} M \geq 2$ and $\operatorname{depth}_{\mathbf{R}} M>0$. Let $Q$ be a parameter ideal for $M$ such that $e_{1}(Q, M)=-\sum_{i=1}^{r-1}\left(\begin{array}{c}r-2 \\ i-1\end{array}\right) h^{i}(M)$. Then $Q \mathrm{H}_{\mathfrak{m}}^{i}(M)=(0)$ for all $1 \leq i \leq r-1$.

For each $x \in \mathfrak{m}$, we put $\mathrm{U}_{M}(x):=\bigcup_{n \geq 0}\left[x M: M \mathfrak{m}^{n}\right]$.

Proposition 5.2. Let $(\mathbf{R}, \mathfrak{m})$ be a Noetherian local ring and $M$ a generalized Cohen-Macaulay $\mathbf{R}$-module with $r=\operatorname{dim}_{\mathbf{R}} M \geq 3$ and $\operatorname{depth}_{\mathbf{R}} M>0$. Let $Q=\left(x_{1}, x_{2}, \ldots, x_{r}\right)$ be a parameter ideal for $M$. Assume that $\left(x_{1}, x_{r}\right) \mathrm{H}_{\mathfrak{m}}^{1}(M)=(0)$ and that the parameter ideal $\left(x_{1}, x_{2}, \ldots, x_{r-1}\right)$ for the generalized Cohen-Macaulay $\mathbf{R}-$ module $M / \mathrm{U}_{M}\left(x_{r}\right)$ is standard. Then $\mathrm{U}_{M}\left(x_{1}\right) \cap Q M=$ $x_{1} M$. 
We then have the following, which is the key in our argument. The proof is similar to the ring case [13, Theorem 2.1] but let us note a brief proof in order to see how we use the previous results Lemma 5.1 and Proposition 5.2 .

Theorem 5.3. Let $(\mathbf{R}, \mathfrak{m})$ be a Noetherian local ring and let $M$ be a generalized CohenMacaulay $\mathbf{R}-$ module with $r=\operatorname{dim}_{\mathbf{R}} M \geq 2$ and $\operatorname{depth}_{\mathbf{R}} M>0$. Let $Q$ be a parameter ideal for $M$. Then the following conditions are equivalent:

(i) $Q$ is a standard parameter ideal for $M$;

(ii) $e_{1}(Q, M)=-\sum_{i=1}^{r-1}\left(\begin{array}{c}r-2 \\ i-1\end{array}\right) h^{i}(M)$.

Proof. We have only to show the implication (ii) $\Rightarrow$ (i). To do this, we may assume that the residue class field $\mathbf{R} / \mathfrak{m}$ of $\mathbf{R}$ is infinite. We write $Q=\left(x_{1}, x_{2}, \ldots, x_{r}\right)$, where each $x_{j}$ is superficial for $M$ with respect to $Q$. Remember that by Lemma $5.1 Q \mathrm{H}_{\mathfrak{m}}^{i}(M)=(0)$ for all $i \neq r$. Hence $Q$ is standard, if $r=2([29$, Corollary 3.7]).

Assume that $r \geq 3$ and that our assertion holds true for $r-1$. Let $1 \leq j \leq r$ be an integer. We put $N=M / x_{j} M, \bar{M}=N / \mathrm{H}_{\mathfrak{m}}^{0}(N)\left(=M / \mathrm{U}_{M}\left(x_{j}\right)\right)$, and $Q_{j}=\left(x_{i} \mid 1 \leq i \leq r, i \neq j\right)$. Then $\mathrm{H}_{\mathfrak{m}}^{i}(N) \cong \mathrm{H}_{\mathfrak{m}}^{i}(\bar{M})$ for all $i \geq 1$. On the other hand, since $x_{j} \mathrm{H}_{\mathfrak{m}}^{i}(M)=(0)$ for $i \neq r$ and $x_{j}$ is $M$-regular, for each $0 \leq i \leq r-2$ we have the short exact sequence

$$
0 \rightarrow \mathrm{H}_{\mathfrak{m}}^{i}(M) \rightarrow \mathrm{H}_{\mathfrak{m}}^{i}(N) \rightarrow \mathrm{H}_{\mathfrak{m}}^{i+1}(M) \rightarrow 0
$$

of local cohomology modules. Hence $\mathbb{I}(M)=\mathbb{I}(N)$ and

$$
\begin{aligned}
e_{1}(Q, M)=e_{1}\left(Q_{j}, N\right) & =e_{1}\left(Q_{j}, \bar{M}\right) \\
& \geq-\sum_{i=1}^{r-2}\left(\begin{array}{c}
r-3 \\
i-1
\end{array}\right) h^{i}(\bar{M}) \\
& =-\sum_{i=1}^{r-2}\left(\begin{array}{c}
r-3 \\
i-1
\end{array}\right) h^{i}(N) \\
& =-\sum_{i=1}^{r-2}\left(\begin{array}{c}
r-3 \\
i-1
\end{array}\right)\left[h^{i}(M)+h^{i+1}(M)\right] \\
& =-\sum_{i=1}^{r-1}\left(\begin{array}{c}
r-2 \\
i-1
\end{array}\right) h^{i}(M) \\
& =e_{1}(Q, M),
\end{aligned}
$$

so that the equality

$$
e_{1}\left(Q_{j}, \bar{M}\right)=-\sum_{i=1}^{r-2}\left(\begin{array}{c}
r-3 \\
i-1
\end{array}\right) h^{i}(\bar{M})
$$

holds for the parameter ideal $Q_{j}$ for the generalized Cohen-Macaulay $\mathbf{R}$-module $\bar{M}=M / \mathrm{U}_{M}\left(x_{j}\right)$. Thus by the hypothesis of induction on $r=\operatorname{dim}_{\mathbf{R}} M, Q_{j}$ is a standard parameter ideal for $M / \mathrm{U}_{M}\left(x_{j}\right)$ for every $1 \leq j \leq r$. Hence $\mathrm{U}_{M}\left(x_{1}\right) \cap Q M=x_{1} M$ by Proposition 5.2. Thus $Q_{1}$ is standard parameter ideal for $M / x_{1} M$ ([29, Corollary 2.3]). Therefore $Q$ is a standard parameter ideal for $M$, because $\mathbb{I}(M)=\mathbb{I}\left(M / x_{1} M\right)$ ([29, Corollary 2.4]).

We are now ready to prove the main result of this section. 
Theorem 5.4. Let $(\mathbf{R}, \mathfrak{m})$ be a Noetherian local ring and $M$ an unmixed $\mathbf{R}$-module with $r=\operatorname{dim}_{\mathbf{R}} M \geq 2$. Then the following conditions are equivalent:

(i) $M$ is a Buchsbaum $\mathbf{R}-$ module;

(ii) The first Hilbert coefficient $e_{1}(Q, M)$ of $M$ is constant and independent of the choice of parameter ideals $Q$ for $M$.

When this is the case, one has the equality

$$
e_{1}(Q, M)=-\sum_{i=1}^{r-1}\left(\begin{array}{l}
r-2 \\
i-1
\end{array}\right) h^{i}(M)
$$

for every parameter ideal $Q$ for $M$, where $h^{i}(M)=\lambda\left(\mathrm{H}_{\mathfrak{m}}^{i}(M)\right)$ for each $1 \leq i \leq r-1$.

Proof. (i) $\Rightarrow$ (ii) This is due to Schenzel [25].

(ii) $\Rightarrow$ (i) Since $\sharp \Lambda(M)=1$, by Proposition $4.2 M$ is a generalized Cohen-Macaulay $\mathbf{R}$ module. Hence $\Lambda(M)=\left\{-\sum_{i=1}^{r-1}\left(\begin{array}{c}r-2 \\ i-1\end{array}\right) h^{i}(M)\right\}$ by [25, Korollar 3.2], so that by Theorem 5.3 every parameter ideal $Q$ for $M$ is standard. Thus $M$ is, by definition, a Buchsbaum $\mathbf{R}$-module $([27])$.

See [25] for the last assertion.

We now in a position to conclude this section with a characterization of $\mathbf{R}$-modules possessing $\sharp \Lambda(M)=1$.

Theorem 5.5. Let $(\mathbf{R}, \mathfrak{m})$ be a Noetherian local ring and $M$ a finitely generated $\mathbf{R}$-module with $r=\operatorname{dim}_{\mathbf{R}} M \geq 2$. Let $U=\mathrm{U}_{\widehat{M}}(0)$ be the unmixed component of $(0)$ in the $\mathfrak{m}$-adic completion $\widehat{M}$ of $M$. Then the following conditions are equivalent:

(i) $\sharp \Lambda(M)=1$;

(ii) $\widehat{M} / U$ is a Buchsbaum $\widehat{\mathbf{R}}-$ module and $\operatorname{dim}_{\widehat{\mathbf{R}}} U \leq r-2$.

When this is the case, one has the equality

$$
e_{1}(Q, M)=-\sum_{i=1}^{r-1}\left(\begin{array}{c}
r-2 \\
i-1
\end{array}\right) h^{i}(\widehat{M} / U)
$$

for every parameter ideal $Q$ for $M$.

Proof. We may assume $\mathbf{R}$ is complete.

(i) $\Rightarrow$ (ii) Since $\sharp \Lambda(M)=1, \operatorname{dim}_{\mathbf{R}} U \leq r-2$ by Proposition 4.3 , We get $\sharp \Lambda(M / U)=1$ by Remark 4.4, so that by Theorem $5.4 M / U$ is a Buchsbaum $\mathbf{R}$-module.

(ii) $\Rightarrow$ (i) We get by Theorem 5.4 that $\sharp \Lambda(M / U)=1$ and hence $\sharp \Lambda(M)=1$ by Lemma 3.3 ,

See Theorem 5.4 for the last assertion.

\section{Homological Degrees}

In this section we deal with the variation of the extended degree function hdeg ([7, [30]), labeled $\operatorname{hdeg}_{I}$ (see [18], [31, p. 142]). We recall the basic properties of these functions. These techniques and their relationships to $e_{1}(I)$ have been mentioned in [32] but the treatment here is more focused. It will lead to sharper bounds in the case of $e_{1}(I, M)$. 
Cohomological degrees. Let $\mathbf{R}$ be a Noetherian local ring with maximal ideal $\mathfrak{m}$ and infinite residue class field. Let $\mathcal{M}(\mathbf{R})$ denote the category of finitely generated $\mathbf{R}$-modules and let $I$ be an $\mathfrak{m}$-primary ideal of $\mathbf{R}$. Then one has the following extension of the classical multiplicity.

Definition 6.1. A cohomological degree, or extended multiplicity function with respect to $I$, is a function

$$
\operatorname{Deg}(\cdot): \mathcal{M}(\mathbf{R}) \rightarrow \mathbb{N}
$$

that satisfies the following conditions. Let $M \in \mathcal{M}(\mathbf{R})$.

(a) If $L=\Gamma_{\mathfrak{m}}(M)$ is the $\mathbf{R}$-submodule of elements of $M$ that are annihilated by a power of the maximal ideal $\mathfrak{m}$ and $\bar{M}=M / L$, then

$$
\operatorname{Deg}(M)=\operatorname{Deg}(\bar{M})+\lambda(L) .
$$

(b) (Bertini's rule) If $M$ has positive depth, then

$$
\operatorname{Deg}(M) \geq \operatorname{Deg}(M / h M)
$$

for every generic hyperplane section $h \in I \backslash \mathfrak{m} I$.

(c) (The calibration rule) If $M$ is a Cohen-Macaulay $\mathbf{R}$-module, then

$$
\operatorname{Deg}(M)=\operatorname{deg}(M)
$$

where $\operatorname{deg}(M)=e_{0}(I, M)$ is the Samuel multiplicity of $M$ with respect to $I$.

The existence of cohomological degrees in arbitrary dimensions was established in [30. Let us formulate it for the case where the $\operatorname{ring} \mathbf{R}$ is complete. The use of the more general Samuel multiplicities was introduced in [18]. When precision demands, we denote the degree and homological degree functions associated to the $\mathfrak{m}$-primary ideal $I$ by $\operatorname{deg}_{I}$ and $\operatorname{hdeg}_{I}$, respectively.

For the rest of this section suppose that $\mathbf{R}$ is complete. For each finitely generated $\mathbf{R}$-module $M$ and $j \in \mathbb{Z}$ let

$$
M_{j}=\operatorname{Hom}_{\mathbf{R}}\left(\mathrm{H}_{\mathfrak{m}}^{j}(M), E\right),
$$

where $E=\mathrm{E}_{\mathbf{R}}(\mathbf{R} / \mathfrak{m})$ denotes the injective envelope of the residue class field. Then, thanks to the local duality theorem, one gets $\operatorname{dim}_{\mathbf{R}} M_{j} \leq j$ for all $j \in \mathbb{Z}$.

Definition 6.2. Let $M$ be a finitely generated $\mathbf{R}$-module with $r=\operatorname{dim}_{\mathbf{R}} M>0$. Then the homological degree of $M$ is the integer

$$
\operatorname{hdeg}(M)=\operatorname{deg}(M)+\sum_{j=0}^{r-1}\left(\begin{array}{c}
r-1 \\
j
\end{array}\right) \cdot \operatorname{hdeg}\left(M_{j}\right) .
$$

We call attention to the fact (see [30] for details) that the notion of generic hyperplane section used for $\operatorname{hdeg}(M)$ are superficial elements for $M$ and for all $M_{j}$, but also for the iterated ones of these modules (there are only a finite number of them).

We will employ hdeg to derive lower bounds for $e_{1}(I, M)$. 
Homological torsion. There are other combinatorial expressions of the terms $\operatorname{hdeg}_{I}\left(M_{j}\right)$ that behave well under hyperplane sections.

Definition 6.3. Let $M$ be an $\mathbf{R}$-module with $r=\operatorname{dim}_{\mathbf{R}} M \geq 2$. For each integer $1 \leq i \leq r-1$ we put

$$
\mathbf{T}_{I}^{(i)}(M)=\sum_{j=1}^{r-i}\left(\begin{array}{c}
r-i-1 \\
j-1
\end{array}\right) \cdot \operatorname{hdeg}_{I}\left(M_{j}\right)
$$

Hence

$$
\operatorname{hdeg}_{I}(M)>\mathbf{T}_{I}^{(1)}(M) \geq \mathbf{T}_{I}^{(2)}(M) \geq \cdots \geq \mathbf{T}_{I}^{(r-1)}(M) .
$$

If $M$ is a generalized Cohen-Macaulay $\mathbf{R}$-module, then

$$
\mathbf{T}_{I}^{(i)}(M)=\sum_{j=1}^{r-i}\left(\begin{array}{c}
r-i-1 \\
j-1
\end{array}\right) \lambda\left(\mathrm{H}_{\mathfrak{m}}^{j}(M)\right)
$$

which is independent of $I$.

We then have the following.

Theorem 6.4. [30, Theorem 2.13] Let $M$ be a finitely generated $\mathbf{R}$-module with $r=\operatorname{dim}_{\mathbf{R}} M$ and let $h$ be a generic hyperplane section. Then $\mathbf{T}_{I}^{(i)}(M / h M) \leq \mathbf{T}_{I}^{(i)}(M)$ for all $1 \leq i \leq r-2$.

We now turn this into a uniform bound for the first Hilbert coefficient of a module $M$ relative to an ideal $I$ generated by a system of parameters of $M$. We note that there are general bounds for all Hilbert coefficients $e_{i}(I, M)$ for arbitrary m-primary ideals $I$ ([22]). Those developed here have a more specialized character and hold only for $e_{1}(I, M)$ and parameter ideals $I$.

Theorem 6.5. Let $M$ be a finitely generated $\mathbf{R}$-module with $\operatorname{dim}_{\mathbf{R}} M=\operatorname{dim} \mathbf{R} \geq 2$ and let $Q$ be a parameter ideal of $\mathbf{R}$. Then

$$
-e_{1}(Q, M) \leq \mathbf{T}_{Q}^{(1)}(M) .
$$

Proof. Let $d=\operatorname{dim} \mathbf{R}$ and let $h \in Q \backslash \mathfrak{m} Q$ be a generic hyperplane section used for $\operatorname{hdeg}_{Q}(M)$. Since

$$
-e_{1}(Q, M)=-e_{1}\left(Q, M / \mathrm{H}_{\mathfrak{m}}^{0}(M)\right) \text { and } \mathbf{T}_{Q}^{(1)}\left(M / \mathrm{H}_{\mathfrak{m}}^{0}(M)\right) \leq \mathbf{T}_{Q}^{(1)}(M),
$$

replacing $M$ with $M / \mathrm{H}_{\mathfrak{m}}^{0}(M)$ if necessary, we may assume $\operatorname{depth}_{\mathbf{R}} M \geq 1$. We may also assume that $h$ is superficial for $M$ and for all $M_{j}(0 \leq j \leq d-1)$ with respect to $Q$. Hence $h$ is $M$-regular and $\lambda\left(M_{1} / h M_{1}\right)<\infty$ (remember that $\left.\operatorname{dim}_{\mathbf{R}} M_{1} \leq 1\right)$. Suppose $d=2$. Then $\mathbf{T}_{Q}^{(1)}(M)=\operatorname{hdeg}_{Q}\left(M_{1}\right)$ and $-e_{1}(Q, M)=\lambda\left((0):_{\mathrm{H}_{\mathfrak{m}}^{1}(M)} h\right)$ by Proposition $2.2(\mathrm{~d})$. On the other hand, from the exact sequence

$$
0 \longrightarrow M \stackrel{h}{\longrightarrow} M \longrightarrow M / h M \longrightarrow 0
$$

of $\mathbf{R}$-modules, we obtain the exact sequence

$$
0 \rightarrow(0):_{\mathrm{H}_{\mathfrak{m}}^{1}(M)} h \rightarrow \mathrm{H}_{\mathfrak{m}}^{1}(M) \stackrel{h}{\rightarrow} \mathrm{H}_{\mathfrak{m}}^{1}(M) .
$$

Then, taking the Matlis dual, we have an epimorphism

$$
M_{1} / h M_{1} \rightarrow \operatorname{Hom}_{\mathbf{R}}\left((0):_{\mathrm{H}_{\mathfrak{m}}^{1}(M)} h, E\right) \rightarrow 0,
$$

so that

$$
\lambda\left((0):_{H_{\mathfrak{m}}^{1}(M)} h\right)=\operatorname{hdeg}_{Q}\left(\operatorname{Hom}_{\mathbf{R}}\left((0):_{\mathrm{H}_{\mathfrak{m}}^{1}(M)} h, E\right)\right) \leq \operatorname{hdeg}_{Q}\left(M_{1} / h M_{1}\right) \leq \operatorname{hdeg}_{Q}\left(M_{1}\right)
$$


by Theorem 6.4. Thus $-e_{1}(Q, M) \leq \mathbf{T}_{Q}^{(1)}(M)$. If $d \geq 3$, then we get

$$
\mathbf{T}_{Q /(h)}^{(1)}(M / h M) \leq \mathbf{T}_{Q}^{(1)}(M)
$$

Hence the result follows, since $-e_{1}(Q, M)=-e_{1}(Q /(h), M / h M)$ by Proposition 2.2 (a).

When the module is generalized Cohen-Macaulay we recover the bound discussed at the beginning of Section 4 .

Corollary 6.6. If $M$ is a generalized Cohen-Macaulay $\mathbf{R}$-module with $\operatorname{dim}_{\mathbf{R}} M \geq 1$, then the Hilbert coefficients $e_{1}(Q, M)$ are bounded for all parameter ideals $Q$ for $M$.

Proof. Passing to the $\operatorname{ring} \mathbf{R} /\left[(0):_{\mathbf{R}} M\right]$, we may assume that $\operatorname{dim} \mathbf{R}=\operatorname{dim}_{\mathbf{R}} M$ and that $Q$ is a parameter ideal of $\mathbf{R}$. Then $e_{1}(Q, M) \leq 0$ by Corollary 2.3. We get by Theorem 6.5 $-e_{1}(Q, M) \leq \mathbf{T}_{Q}^{(1)}(M)$, while $\mathbf{T}_{Q}^{(1)}(M)=\sum_{j=1}^{d-1}\left(\begin{array}{c}d-2 \\ j-1\end{array}\right) \lambda\left(\mathrm{H}_{\mathfrak{m}}^{j}(M)\right)$ is independent of the choice of $Q$. Hence the result.

Corollary 6.7. Suppose that $\operatorname{dim}_{\mathbf{R}} M \geq 1$. Then the set $\left\{e_{1}(Q, M) \mid Q\right.$ are parameter ideals of $M$ with the same integral closure $\}$ is finite.

Proof. For each parameter ideal $Q$ of $M$ we get $e_{1}(Q, M) \leq 0$, while Theorem 6.5 asserts that $e_{1}(Q, M) \geq-\mathbf{T}_{Q}^{(1)}(M)$. Hence the result follows, because $\mathbf{T}_{Q}^{(1)}(M)$ depends only on $\bar{Q}$, the integral closure of $Q$.

\section{Euler CHARACteristics ANd Hilbert CHARACTERISTiCs}

The relationship between partial Euler characteristics and superficial elements make for a straightforward comparison with extended degree functions. Unless otherwise specified, throughout it is assumed that $\mathbf{R}$ is a Noetherian complete local ring with infinite residue class field. We will prove that Euler characteristics can be uniformly bounded by homological degrees. The basic tool is the following observation, which is found in the proof of [3, Theorem 4.6.10 (a)].

Proposition 7.1. Let $M$ be a finitely generated $\mathbf{R}$-module with $r=\operatorname{dim}_{\mathbf{R}} M \geq 2$. Let $\mathbf{x}=$ $\left\{x_{1}, x_{2}, \ldots, x_{r}\right\}$ be a system of parameters for $M$ and set $\mathbf{x}^{\prime}=\left\{x_{2}, \ldots, x_{r}\right\}$. Then

$$
\chi_{1}(\mathbf{x} ; M)=\chi_{1}\left(\mathbf{x}^{\prime} ; M / x_{1} M\right)+\chi_{1}\left(\mathbf{x}^{\prime} ; 0:_{M} x_{1}\right) .
$$

Theorem 7.2. Let $M$ be a finitely generated $\mathbf{R}$-module with $\operatorname{dim}_{\mathbf{R}} M=\operatorname{dim} \mathbf{R}=d \geq 1$. Then for every system $\mathbf{x}=\left\{x_{1}, x_{2}, \ldots, x_{d}\right\}$ of parameters of $\mathbf{R}$, one has

$$
\chi_{1}(\mathbf{x} ; M) \leq \operatorname{hdeg}_{Q}(M)-\operatorname{deg}_{Q}(M)
$$

where $Q=(\mathbf{x})$.

Proof. As $\lambda(M / Q M)=\chi_{1}(\mathbf{x} ; M)+\operatorname{deg}_{Q}(M)$, we have only to show $\lambda(M / Q M) \leq \operatorname{hdeg}_{Q}(M)$. Let $h \in Q \backslash \mathfrak{m} Q$ be a generic hyperplane section used for $\operatorname{hdeg}_{Q}(M)$. Then, since $\lambda(M / Q M)=$ $\lambda((M / h M) / Q \cdot(M / h M))$ and $\operatorname{hdeg}_{Q /(h)}(M / h M) \leq \operatorname{hdeg}_{Q}(M)$, by induction on $d$ we may assume $d=1$. When $d=1, \chi_{1}(\mathbf{x} ; M)=\lambda\left(0:_{M} x_{1}\right)$ and hence $\lambda(M / Q M)=\chi_{1}(\mathbf{x} ; M)+$ $\operatorname{deg}_{Q}(M) \leq \lambda\left(\mathrm{H}_{\mathfrak{m}}^{0}(M)\right)+\operatorname{deg}_{Q}(M)=\operatorname{hdeg}_{Q}(M)$, as wanted. 
Corollary 7.3. Suppose that $\operatorname{dim}_{\mathbf{R}} M \geq 1$. Then for every primary ideal I of $M$, the set

$$
\Xi_{I}(M)=\left\{\chi_{1}(\mathbf{x}, M) \mid \mathbf{x} \text { are systems of parameters of } \mathrm{M} \text { with } \overline{(\mathbf{x})}=\bar{I}\right\}
$$

is finite.

Proof. Both $\operatorname{hdeg}_{Q}(M)$ and $\operatorname{deg}_{Q}(M)$ depend only on the integral closure $\bar{I}=\bar{Q}$ of $Q=(\mathbf{x})$.

Definition 7.4. Let $\mathbf{R}$ be a Noetherian local ring and $M$ a finitely generated $\mathbf{R}$-module with $r=\operatorname{dim}_{\mathbf{R}} M \geq 1$. For each system $\mathbf{x}=\left\{x_{1}, x_{2}, \ldots, x_{r}\right\}$ of parameters of $M$, the Hilbert characteristic of $M$ with respect to $Q=(\mathbf{x})$ is defined to be

$$
\mathbf{h}(\mathbf{x} ; M)=\sum_{i=0}^{r}(-1)^{i} e_{i}(Q, M) .
$$

The following proposition shows that the Hilbert characteristic can be characterized as a quasi-cohomological degree for $M$.

Proposition 7.5. Let $(\mathbf{R}, \mathfrak{m})$ be a Noetherian local ring and $M$ a finitely generated $\mathbf{R}$-module with $r=\operatorname{dim}_{\mathbf{R}} M \geq 1$. Let $\mathbf{x}=\left\{x_{1}, x_{2}, \ldots, x_{r}\right\}$ be a system of parameters of $M$ and a $d-$ sequence for $M$. Then the Hilbert characteristic of $M$ with respect to $\mathbf{x}$ satisfies the following.

(a) Suppose that $x_{1}$ is a superficial element for $M$ and $\operatorname{depth}_{\mathbf{R}} M \geq 1$. Then

$$
\mathbf{h}(\mathbf{x} ; M)=\mathbf{h}\left(\mathbf{x}^{\prime} ; M / x_{1} M\right),
$$

where $\mathbf{x}^{\prime}=\left\{x_{2}, \ldots, x_{r}\right\}$.

(b) Let $M_{0}=\mathrm{H}_{\mathfrak{m}}^{0}(M)$ and $M^{\prime}=M / M_{0}$. Then

$$
\mathbf{h}(\mathbf{x} ; M)=\mathbf{h}\left(\mathbf{x} ; M^{\prime}\right)+\lambda\left(M_{0}\right) .
$$

Proof. Let $Q=(\mathbf{x})$. Recall that, by [14, Proposition 3.4], we have $(-1)^{r} e_{r}(Q, M)=\lambda\left(H_{\mathfrak{m}}^{0}(M)\right)$.

(a) We may assume that $x_{1}$ is $M$-regular. By Proposition 2.2 , we obtain

$$
\mathbf{h}(\mathbf{x} ; M)=\sum_{i=0}^{r-1}(-1)^{i} e_{i}(Q, M)+(-1)^{r} e_{r}(Q, M)=\sum_{i=0}^{r-1}(-1)^{i} e_{i}\left(\mathbf{x}^{\prime}, M / x_{1} M\right)=\mathbf{h}\left(\mathbf{x}^{\prime} ; M / x_{1} M\right)
$$

(b) By applying Proposition 2.2 (b) to the exact sequence $0 \rightarrow M_{0} \rightarrow M \rightarrow M^{\prime} \rightarrow 0$, we get

$$
e_{i}(Q, M)=e_{i}\left(Q, M^{\prime}\right) \quad \text { for all } 0 \leq i \leq r-1 \text {. }
$$


Note that $(-1)^{r} e_{r}\left(Q, M^{\prime}\right)=\lambda\left(H_{\mathfrak{m}}^{0}\left(M^{\prime}\right)\right)=0$. Hence

$$
\begin{aligned}
\mathbf{h}(\mathbf{x} ; M)=\sum_{i=0}^{r}(-1)^{i} e_{i}(Q, M) & =\sum_{i=0}^{r-1}(-1)^{i} e_{i}(Q, M)+\lambda\left(M_{0}\right) \\
& =\sum_{i=0}^{r-1}(-1)^{i} e_{i}\left(Q, M^{\prime}\right)+\lambda\left(M_{0}\right) \\
& =\sum_{i=0}^{r}(-1)^{i} e_{i}\left(Q, M^{\prime}\right)+\lambda\left(M_{0}\right) \\
& =\mathbf{h}\left(\mathbf{x} ; M^{\prime}\right)+\lambda\left(M_{0}\right) .
\end{aligned}
$$

Proposition 7.6. Let $(\mathbf{R}, \mathfrak{m})$ be a Noetherian local ring and $M$ a finitely generated $\mathbf{R}$-module with $r=\operatorname{dim}_{\mathbf{R}} M \geq 1$. Let $\mathbf{x}=\left\{x_{1}, x_{2}, \ldots, x_{r}\right\}$ be a system of parameters of $M$ and $a$ $d$-sequence for $M$. Let $Q=(\mathbf{x})$. Then

$$
\mathbf{h}(\mathbf{x} ; M)=\lambda(M / Q M) .
$$

In particular, $\mathbf{h}(\mathbf{x} ; M) \geq e_{0}(Q, M)$ with equality if and only if $M$ is Cohen-Macaulay.

Proof. Using [10, Theorem 3.7], one can prove that

$$
(-1)^{i} e_{i}(Q, M)=\chi_{1}\left(x_{1}, \ldots, x_{r-i}, x_{r-i+1} ; M\right)-\chi_{1}\left(x_{1}, \ldots, x_{r-i} ; M\right) \geq 0
$$

for all $1 \leq i \leq r$. Therefore

$$
\begin{aligned}
\mathbf{h}(\mathbf{x} ; M) & =e_{0}(Q, M)+\sum_{i=1}^{r}(-1)^{i} e_{i}(Q, M) \\
& =e_{0}(Q, M)+\sum_{i=1}^{r}\left(\chi_{1}\left(x_{1}, \ldots, x_{r-i}, x_{r-i+1} ; M\right)-\chi_{1}\left(x_{1}, \ldots, x_{r-i} ; M\right)\right) \\
& =\chi_{0}(\mathbf{x} ; M)+\chi_{1}(\mathbf{x} ; M) \\
& =\lambda(M / Q M) .
\end{aligned}
$$

Corollary 7.7. Let $\mathbf{x}$ be a system of parameters of $M$ which is a d-sequence for $M$. Suppose that $\mathbf{x} \in \mathfrak{m} \backslash \mathfrak{m}^{2}$. Then the Betti numbers $\beta_{i}^{\mathbf{R}}(M)$ satisfy

$$
\beta_{i}^{\mathbf{R}}(M) \leq \lambda(M /(\mathbf{x}) M) \cdot \beta_{i}^{\mathbf{R}}(k) .
$$

Proof. It follows from the argument of [31, Theorem 2.94], where we use the properties of $\mathbf{h}(\mathbf{x} ; M)$ in the induction part.

Remark 7.8. Note that the condition $\mathbf{x} \in \mathfrak{m} \backslash \mathfrak{m}^{2}$ in Corollary 7.7 is needed in the induction argument which requires the inequality of Betti numbers $\beta_{i}^{\mathbf{R} /\left(x_{1}\right)}(k) \leq \beta_{i}^{\mathbf{R}}(k)([15$, Corollary 3.4.2]). 


\section{Buchsbaum-Rim Coefficients}

In this section let us note another set of related questions, concerned about the vanishing and the negativity of the Buchsbaum-Rim coefficients of modules.

Let $\mathbf{R}$ be a Noetherian local ring with maximal ideal $\mathfrak{m}$ and $d=\operatorname{dim} \mathbf{R} \geq 1$. The BuchsbaumRim multiplicity (4]) arises in the context of an embedding

$$
0 \rightarrow E \longrightarrow F=\mathbf{R}^{s} \longrightarrow C \rightarrow 0
$$

of $\mathbf{R}$-modules, where $E \subseteq \mathfrak{m} F$ and $C$ has finite length. Let

$$
\varphi: \mathbf{R}^{m} \longrightarrow F=\mathbf{R}^{s}
$$

be an $\mathbf{R}$-linear map represented by a matrix with entries in $\mathfrak{m}$ such that $\operatorname{Im} \varphi=E$. We then have a homomorphism

$$
\mathcal{S}(\varphi): \mathcal{S}\left(\mathbf{R}^{m}\right) \longrightarrow \mathcal{S}\left(\mathbf{R}^{s}\right)
$$

of symmetric algebras, whose image is the Rees algebra $\mathcal{R}(E)$ of $E$, and whose cokernel we denote by $C(\varphi)$. Hence

$$
0 \rightarrow \mathcal{R}(E) \longrightarrow \mathcal{S}\left(\mathbf{R}^{s}\right)=\mathbf{R}\left[T_{1}, T_{2}, \ldots, T_{s}\right] \longrightarrow C(\varphi) \rightarrow 0 .
$$

This exact sequence (with a different notation) is studied in [4 in great detail. Of significance for us is the fact that $C(\varphi)$, with the grading induced by the homogeneous homomorphism $S(\varphi)$, has components of finite length, for which we have the following. Let $E^{n}=[\mathcal{R}(E)]_{n}$ and $F^{n}=[\mathcal{S}(F)]_{n}$ for $n \geq 0$, where $F=\mathbf{R}^{s}$.

Theorem 8.1. $\lambda\left(F^{n} / E^{n}\right)$ is a polynomial in $n$ of degree $d+s-1$ for $n \gg 0$ :

$$
\lambda\left(F^{n} / E^{n}\right)=\operatorname{br}(E)\left(\begin{array}{c}
n+d+s-2 \\
d+s-1
\end{array}\right)-\operatorname{br}_{1}(E)\left(\begin{array}{c}
n+d+s-3 \\
d+s-2
\end{array}\right)+\text { lower terms. }
$$

This polynomial is called the Buchsbaum-Rim polynomial of $E$. The leading coefficient $\operatorname{br}(E)$ is the Buchsbaum-Rim multiplicity of $\varphi$; if the homomorphism $\varphi$ is understood, we shall simply denote it by $\operatorname{br}(E)$. This number is determined by an Euler characteristic of the Buchsbaum-Rim complex ([4]).

Assume now the residue class field of $\mathbf{R}$ is infinite. The minimal reductions $U$ of $E$ are generated by $d+s-1$ elements. We refer to $U$ as a parameter module of $F$. The corresponding coefficients are $\operatorname{br}(U)=\operatorname{br}(E)$ but $\operatorname{br}_{1}(U) \leq \operatorname{br}_{1}(E)$. It is not clear what the possible values of $\mathrm{br}_{1}(U)$ are, and in similarity to the case of ideals, we can ask the following.

(a) $\operatorname{br}_{1}(U) \leq 0$ ?

(b) Suppose that $\mathbf{R}$ is unmixed. Then is $\mathbf{R}$ Cohen-Macaulay, if $\operatorname{br}_{1}(U)=0$ ?

(c) Are the values of $\operatorname{br}_{1}(U)$ bounded?

(d) What happens in low dimensions?

As for question (a), a surprising result of Hayasaka and Hyry shows the negativity of $\operatorname{br}_{1}(U)$ in the following way. It gives an eminent proof of Corollary 2.3.

Theorem 8.2 ([16, Theorem 1.1]). $\lambda\left(F^{n} / U^{n}\right) \geq \operatorname{br}(U)\left(\begin{array}{c}n+d+s-2 \\ d+s-1\end{array}\right)$ for all $n \geq 0$. Hence

$$
\mathrm{br}_{1}(U) \leq 0 \text {. }
$$


They also proved that $\mathbf{R}$ is a Cohen-Macaulay ring, once $\lambda\left(F^{n} / U^{n}\right)=\operatorname{br}(U)\left(\begin{array}{c}n+d+s-2 \\ d+s-1\end{array}\right)$ for some $n \geq 1$. When this is the case, one has the equality $\lambda\left(F^{n} / U^{n}\right)=\operatorname{br}(U)\left(\begin{array}{c}n+d+s-2 \\ d+s-1\end{array}\right)$ for all $n \geq 0$, whence $\operatorname{br}_{1}(U)=0$ ([2, Theorem 3.4]).

Note that question (c) is answered affirmatively for $s=1$ in Corollary 6.7.

We close this paper with the following.

Conjecture 8.3. Let $(\mathbf{R}, \mathfrak{m})$ be a Noetherian local $\operatorname{ring}$ with $\operatorname{dim} \mathbf{R} \geq 2$ and let $U \subseteq \mathfrak{m} \mathbf{R}^{s}$ be a parameter module of $\mathbf{R}^{s}(s>0)$. Then $\mathbf{R}$ is a Cohen-Macaulay ring if and only if $\mathbf{R}$ is unmixed and $\operatorname{br}_{1}(U)=0$.

\section{REFERENCES}

[1] M. Auslander and D. Buchsbaum, Codimension and multiplicity, Ann. Math. 68 (1958), 625-657.

[2] J. Brennan, B. Ulrich and W. V. Vasconcelos, The Buchsbaum-Rim polynomial of a module, J. Algebra 241 (2001), 379-392.

[3] W. Bruns and J. Herzog, Cohen-Macaulay Rings, Cambridge University Press, 1993.

[4] D. Buchsbaum and D. S. Rim, A generalized Koszul complex II. Depth and multiplicity, Trans. Amer. Math. Soc. 111 (1965), 197-224.

[5] N. T. Cuong, $p$-standard system of parameters and $p$-standard ideals in local rings, Acta Mathematica Vietnamica 20 (1995), 145-161.

[6] N. T. Cuong, P. Schenzel and N. V. Trung, Verallgemeinerte Cohen-Macaulay-Moduln, Math. Nachr. 85 (1978), 57-73.

[7] L. R. Doering, T. Gunston and W. V. Vasconcelos, Cohomological degrees and Hilbert functions of graded modules, American J. Math. 120 (1998), 493-504.

[8] L. Ghezzi, S. Goto, J. Hong, K. Ozeki, T. T. Phuong and W. V. Vasconcelos, Cohen-Macaulayness versus the vanishing of the first Hilbert coefficient of parameter ideals, J. London Math. Soc. 81 (2010), 679-695.

[9] L. Ghezzi, J. Hong and W. V. Vasconcelos, The signature of the Chern coefficients of local rings, Math. Research Letters 16 (2009), 279-289.

[10] S. Goto, J. Hong and W. V. Vasconcelos, The homology of parameter ideals, J. Algebra 368 (2012), 271-299.

[11] S. Goto and Y. Nakamura, Multiplicities and tight closures of parameters, J. Algebra 244 (2001), 302-311.

[12] S. Goto and K. Nishida, Hilbert coefficients and Buchsbaumness of associated graded rings, J. Pure and Appl. Algebra 181 (2003), 61-74.

[13] S. Goto and K. Ozeki, Buchsbaumness in local rings possessing constant first Hilbert coefficient of parameters, Nagoya Math. J. 199 (2010), 95-105.

[14] S. Goto and K. Ozeki, Uniform bounds for Hilbert coefficients of parameters, in Commutative Algebra and its Connections to Geometry, 97-118, Contemp. Math. 555, Amer. Math. Soc., Providence, RI, 2011.

[15] T. Gulliksen and G. Levin, Homology of local rings, Queen's Paper in Pure and Applied Mathematics, No. 20 Queen's University, Kingston, Ont. 1969.

[16] F. Hayasaka and E. Hyry, On the Buchsbaum-Rim function of a parameter module, J. Algebra 327 (2011), 307-315.

[17] T. Kawasaki, On Cohen-Macaulayfication of certain quasi-projective schemes, J. Math. Soc. Japan 50 (1998), 969-991.

[18] C. H. Linh, Upper bound for the Castelnuovo-Mumford regularity of associated graded modules, Comm. Algebra 33 (2005), 1817-1831.

[19] M. Mandal, B. Singh and J. K. Verma, On some conjectures about the Chern numbers of filtrations, J. Algebra 325 (2011), 147-162.

[20] H. Matsumura, Commutative Algebra, Benjamin/Cummings, Reading, 1980.

[21] M. Nagata, Local Rings, Interscience, New York, 1962.

[22] M. E. Rossi, N. V. Trung and G. Valla, Castelnuovo-Mumford regularity and extended degree, Trans. Amer. Math. Soc. 355 (2003), 1773-1786.

[23] M. E. Rossi and G. Valla, On the Chern number of a filtration, Rend. Semin. Mat. Univ. Padova 121 (2009), 201-222. 
[24] M. E. Rossi and G. Valla, Hilbert Functions of Filtered Modules, Lecture Notes of the Unione Matematica Italiana 9, Springer-Verlag, Berlin; UMI, Bologna, 2010.

[25] P. Schenzel, Multiplizitäten in verallgemeinerten Cohen-Macaulay-Moduln, Math. Nachr. 88 (1979), 295306.

[26] J.-P. Serre, Algèbre Locale. Multiplicités, Lecture Notes in Mathematics 11, Springer, Berlin, 1965.

[27] J. Stückrad and W. Vogel, Toward a theory of Buchsbaum singularities, Amer. J. Math. 100 (1978), $727-746$.

[28] J. Stückrad and W. Vogel, Buchsbaum Rings and Applications, Springer, Berlin, 1986.

[29] N. V. Trung, Toward a theory of generalized Cohen-Macaulay modules, Nagoya Math. J. 102 (1986), 1-49.

[30] W. V. Vasconcelos, The homological degree of a module, Trans. Amer. Math. Soc. 350 (1998), $1167-1179$.

[31] W. V. Vasconcelos, Integral Closure, Springer Monographs in Mathematics, New York, 2005.

[32] W. V. Vasconcelos, The Chern coefficients of local rings, Michigan Math. J. 57 (2008), 725-743.

Department of Mathematics, New York City College of Technology-Cuny, 300 Jay Street, BroOKLYN, NY 11201, U. S. A.

E-mail address: lghezzi@citytech.cuny.edu

Department of Mathematics, School of Science and Technology, Meiji University, 1-1-1 HigashiMITA, TAMA-KU, KAWASAKI 214-8571, JAPAN

E-mail address: goto@math.meiji.ac.jp

Department of Mathematics, Southern Connecticut State University, 501 Crescent Street, New Haven, CT 06515-1533, U. S. A.

E-mail address: hongj2@southernct.edu

Department of Mathematics, School of Science and Technology, Meiji University, 1-1-1 HigashiMITA, TAMA-KU, KAWASAKI 214-8571, JAPAN

E-mail address: kozeki@math.meiji.ac.jp

Department of Information Technology and Applied Mathematics, Ton Duc Thang University, 98 Ngo Tat To Street, Ward 19, Binh Thanh District, Ho Chi Minh City, Vietnam

E-mail address: sugarphuong@gmail.com

Department of Mathematics, Rutgers University, 110 Frelinghuysen Rd, Piscataway, NJ 088548019, U. S. A.

E-mail address: vasconce@math.rutgers.edu 